\title{
On the formula of Jacques-Louis Lions for reproducing kernels of harmonic and other functions
}

\author{
By Miroslav Engliš at Prague, Dag Lukkassen at Narvik, Jaak Peetre at Lund, \\ and Lars-Erik Persson at Luleå
}

\begin{abstract}
We give a simpler proof of the formula, due to J.-L. Lions, for the reproducing kernel of the space of harmonic functions on a domain $\Omega \subset \mathbb{R}^{n}$ whose boundary values belong to the Sobolev space $H^{s}(\partial \Omega)$, and also obtain generalizations of this formula when instead of harmonic functions one considers functions annihilated by a given elliptic partial differential operator. Further, we compute the reproducing kernels explicitly in several examples, which leads to an occurrence of new special functions. Some spaces of caloric functions are also briefly considered.
\end{abstract}

\section{Introduction}

Jacques-Louis Lions [L2] (see also [L1]) found a remarkable formula for the reproducing kernel of some function spaces. In fact, Lions died on May 17, 2001, so this was one of his last theorems. ${ }^{1)}$

Let us, for the Reader's benefit, begin by briefly recalling some salient facts about reproducing kernels in general. No previous knowledge of the subject is required, so we hope that this Introduction may be read not only as an introduction to the theme of Lions's formula but may also be viewed as an independent essay on reproducing kernels.

Let $H$ be an arbitrary Hilbert space of real continuous functions on some space $\Omega$, and assume that all point evaluations $\Omega \ni b \mapsto u(b) \in \mathbb{R}$ are continuous, for any $u \in H$. Then it follows from the F. Riesz lemma that for any $b \in \Omega$ there exists an element $K_{b} \in H$ such that

The first author was supported by GA AV ČR grant no. A1019005.

The third author was supported in part by DGI (BFM2001-1424) and by Kungl. Fysiografiska Sällskapet $\mathrm{i}$ Lund (Walter Gyllenbergs fond).

1) Lions was from the onset the Honorary President of the Lund conference. On December 14, 2000 he also submitted the paper [L2] to its proceedings. But on February 12, 2001 an electronic message was received from the secretary of Lions giving the information that he was sick and could not work as usual. 


$$
u(b)=\left(u, K_{b}\right)_{H} .
$$

It is convenient to put $K(x, b)=K_{b}(x)$; this function of two arguments $x, b \in \Omega$ is usually called the reproducing kernel of $H$. Plugging in $u=K_{a}$, with $u(b)=K(b, a)$, into (1.1) we get the important formula

$$
K(b, a)=\left(K_{a}, K_{b}\right)_{H} .
$$

In particular, putting $a=b$ in (1.2), we obtain

$$
K(a, a)=\left\|K_{a}\right\|_{H}^{2} .
$$

Another consequence of (1.1) is the inequality

$$
\operatorname{det}\left(K\left(a_{i}, a_{k}\right)\right)_{1 \leqq i, k \leqq r} \geqq 0,
$$

where $a_{1}, \ldots, a_{r}$ is any finite set of points in $\Omega$. (For the proof consider any linear combination $\sum_{i=1}^{r} c_{i} K_{a_{i}}$ and use (1.2).) Conversely, given any function $K(x, b)$ satisfying (1.4), then there is a Hilbert space $H$ whose reproducing kernel is $K(x, b)$. This theorem was established by Aronszajn [A].

Remark 1.1. In quantum physics reproducing kernels are known as coherent states. These may be viewed as spatially concentrated bunches of energy and, supposedly, their existence has been experimentally verified. One can also reverse the order of things and start with a map from $\Omega$ into an (abstract) Hilbert space $H: \Omega \ni a \mapsto K_{a} \in H$. Then one can view the elements of $H$ as functions on $\Omega$ taking (1.1) as the definition of point evaluations.

Next, by Schwarz's inequality we obtain from (1.1)

$$
|u(b)| \leqq\|u\|_{H}\left\|K_{b}\right\|_{H},
$$

with equality if and only if $u$ is proportional to $K_{b}$. It follows that the reproducing kernel has the following variational description (cf. [L2], (3.1)-(3.3)): The minimum problem

$$
\inf _{u}\|u\|_{H}^{2}, \quad u(b)=1,
$$

has for any given point $b \in \Omega$ the unique solution $w$ given by

$$
w=\frac{1}{\left\|K_{b}\right\|_{H}^{2}} K_{b}
$$

or

$$
w(x)=\frac{1}{\left\|K_{b}\right\|_{H}^{2}} K(x, b) \quad(x \in \Omega) .
$$


Here are some references on reproducing kernels: $[\mathrm{A}],[\mathrm{B}],[\mathrm{G} 1],[\mathrm{Sa}],[\mathrm{Schi}],[\mathrm{Schw}]$, $[\mathrm{Sz}],[\mathrm{Z} 1],[\mathrm{Z} 2]$ (some of them also quoted in [L2]). [B] is a classic, however mostly dealing with Hilbert spaces of holomorphic functions. Another is the book [BS], of which Part II is devoted to Hilbert spaces of solutions of second order elliptic differential operators in planar domains $\Omega$, of the special type $L=-\Delta+q$, where the function $q$ is positive in $\Omega$. A rather recent book is $[\mathrm{Sa}]$. In Chap. I, Section 1 there a sketch of the historical development is given, giving some further references. In particular, its author evokes names such as $\mathbf{J}$. Mercer and E. H. Moore, today perhaps fallen into oblivion. holds:

Next pick an orthonormal basis $\left\{e_{v}\right\}(v=1,2, \ldots)$ in $H$. Then the following formula

$$
K(x, b)=\sum_{v} e_{v}(x) e_{v}(b)
$$

Proof of (1.8). In the formula (1.1) take successively $u=e_{v}(v=1,2, \ldots)$.

Remark 1.2. In the complex case formula (1.8) must be modified in such a way that the second factor $e_{v}(b)$ is replaced by its complex conjugate $\overline{e_{v}(b)}$. The proof is the same as above. Note that (1.1) holds unchanged in this case.

Next, having told all that we need to know about reproducing kernels in general, let us also briefly recall what Lions's formula is about. As in [L2], let $\Omega$ be a bounded domain in $\mathbb{R}^{n}$ with smooth boundary $\Gamma .^{2)}$

We consider the vector space $\mathscr{H}^{s}(\Omega)$ of harmonic distributions $u$ in $\Omega(\Delta u=0)$ which admit a continuation as distributions to a neighborhood of $\bar{\Omega}$ and the trace $\gamma u$ of which on $\Gamma$ belongs to the usual Sobolev space $H^{s}(\Gamma) .{ }^{3)}$ (The existence of this trace in these hypotheses is, incidentally, a consequence of [Pe1], Théorème 1 in Chapitre VI, §1!)

Let $\Lambda$ be the negative of the Laplace-Beltrami operator ${ }^{4)}$ of $\Gamma$ (i.e. $\Lambda=-\Delta_{\Gamma}$ ).

2) It ought to be possible to extend Lions's formula also to suitable unbounded domains! So far we have however no general result. We do not even know if there exists a sufficiently general theory of elliptic boundary problems worked out for unbounded domains.

3) Sobolev spaces can be defined for any compact manifold and for some non-compact ones as well. S. L. Sobolev himself defined, in the mid 1930's, also non-Hilbertian spaces based on an $L_{p}$-metric $(1 \leqq p \leqq \infty)$; these are often denoted $W_{p}^{m}, m$ integer $\geqq 0$. In the 1950's the scale of Hilbert spaces $H^{s}$ (the case $p=2, s=m$ integer) became immensely popular (see [Pe1], [Pe2], [H1], [H2]). One defines first $H^{s}$ for $\mathbb{R}^{n}$ and then, exploiting the invariance for change of local coordinates, patches together local coordinate manifolds to obtain the global space.

4) The Laplace-Beltrami operator is defined on an arbitrary Riemannian manifold with the metric $d s^{2}=\sum_{j, k} g_{j k} d x_{j} d x_{k}$ by the formula

$$
\Delta_{\Gamma}=\frac{1}{\sqrt{g}} \sum_{j, k} \frac{\partial}{\partial x_{j}} \sqrt{g} g^{j k} \frac{\partial}{\partial x_{k}}
$$

where $g^{j k}$ is the matrix inverse to $g_{j k}$ and $g$ the determinant of the latter. If $\Gamma$ is the boundary of a domain in $\mathbb{R}^{n}$, as in our case, one takes the metric induced by the Euclidean metric of the ambient space. In this case it is also possible to give a simple interpretation of the Laplace-Beltrami operator as follows. If a function $f$ on $\Gamma$ is given, extend it to a small neighbourhood of $\Gamma$ in $\mathbb{R}^{n}$ by making it constant in the directions of the normals to $\Gamma$. Then apply the ordinary (Euclidean) Laplacian to this extended function, and take restriction back to $\Gamma$. The result is $\Delta_{\Gamma} f$. 
We need also the differential operator $M$ given by

$$
M=\Lambda+1
$$

(We may refer to $M$ as the smoothed Laplace-Beltrami operator of $\Gamma$.) Then we can make $H^{s}(\Gamma)$ into a Hilbert space in a natural way: Let $L^{2}(\Gamma)$ be the Hilbert space of square integrable functions on $\Gamma$ with respect to the $(n-1)$-dimensional measure $\sigma$ on $\Gamma$. Viewing $M$ as a positive self-adjoint operator in $L^{2}(\Gamma)$ (so that it makes sense to speak of its powers of any fractional order), we equip the latter with the operator norm of $M^{\frac{s}{2}}$ :

$$
\|u\|_{s}=\|u\|_{H^{s}(\Gamma)}=\left\|M^{\frac{s}{2}} u\right\|_{L^{2}(\Gamma)}
$$

and the associated inner product

$$
(u, v)_{s}=(u, v)_{H^{s}(\Gamma)}=\left(M^{\frac{s}{2}} u, M^{\frac{s}{2}} v\right)_{L^{2}(\Gamma)}=\left(M^{s} u, v\right)_{L^{2}(\Gamma)} .
$$

Accordingly, we then equip $\mathscr{H}^{s}(\Omega)$ with the induced norm and inner product

$$
\|u\|_{\mathscr{H}^{s}(\Omega)}:=\|\gamma u\|_{H^{s}(\Gamma)}, \quad(u, v)_{\mathscr{H}^{s}(\Omega)}:=(\gamma u, \gamma v)_{H^{s}(\Gamma)},
$$

respectively.

Remark 1.3. In the manuscript of [L2], the author did use only the operator $\Lambda$ itself. However, as $\Lambda$ is not invertible it is not possible to define negative powers of $\Lambda$. The switch to the smoothed operator $M$ was performed by the editors of the book containing [L2]. (The same oversight occurs also in $[\mathrm{LM}]$, p. 42. In a letter directed to one of us Professor Magenes has admitted this.) Note also that Lions used, instead of our $s$, a twice as large quantity, to wit the parameter $S=s / 2$ or $s=2 S$. Our $s$ measures the order of differentiation or smoothness.

Remark 1.4. The most natural instance of the space $\mathscr{H}^{s}(\Omega)$ is the case $s=0$, that is, the space $\mathscr{H}^{0}(\Omega)$. Thus the elements of $\mathscr{H}^{0}(\Omega)$ are, roughly speaking, harmonic functions whose trace belongs to $L^{2}(\Gamma)$.

In order to obtain an orthonormal basis in $\mathscr{H}^{s}(\Omega)$ we take first an orthonormal basis in $L^{2}(\Gamma)$ consisting of eigenfunctions $e_{v}$ of $M$ so that

$$
\left(e_{v}, e_{\mu}\right)_{L^{2}(\Gamma)}=\delta_{v \mu} \quad \text { and } \quad M e_{v}=\lambda_{v} e_{v} ; \quad M^{s} e_{v}=\lambda_{v}^{s} e_{v},
$$

where $\lambda_{v}$ are the eigenvalues of $M$ (each eigenvalue enters then precisely as many times as its multiplicity indicates). We observe now that

$$
\left(e_{v}, e_{\mu}\right)_{H^{s}(\Gamma)}=\left(M^{s} e_{v}, e_{\mu}\right)_{L^{2}(\Gamma)}=\left(\lambda_{v}^{s} e_{v}, e_{\mu}\right)_{L^{2}(\Gamma)}=\delta_{v \mu} \lambda_{v}^{s}
$$

If we set

$$
\hat{e}_{v}=\lambda_{v}^{-\frac{s}{2}} e_{v}
$$

we obtain thus an orthonormal basis in $H^{s}(\Gamma)$. For simplicity, we will denote by the same 
symbols $e_{v}, \hat{e}_{v}$, etc., also the harmonic or Poisson extension of these functions into the interior of $\Omega$. Then it follows from (1.8) that

$$
K(x, b)=\sum_{v} \hat{e}_{v}(x) \hat{e}_{v}(b)=\sum_{v} \lambda_{v}^{-s} e_{v}(x) e_{v}(b)
$$

We observe that (1.10) involves a zeta function of the Minakshisundaram-Pleijel type [MP].

Let $G(x, b)$ be the Green function of $\Omega$ (that is, writing $G_{b}(x)=G(x, b)$, one has $\left.\Delta G_{b}=\delta_{b}\right)$. Note that

$$
P_{x}(y):=P(y, x):=N_{y} G(y, x)=N_{y} G(x, y) \quad(x \in \Omega, y \in \Gamma)
$$

is the Poisson kernel. Here $N$ is the exterior normal to $\Gamma$, and we let at the same time $N$ denote differentiation in the direction of the normal, in symbols: $N u=\frac{\partial u}{\partial N}$, for any function $u{ }^{5)}$ We may refer to $P(y, \cdot)$ as the Poisson kernel with pole or singularity at $y \in \Gamma$; it can be defined as the solution of a distributional boundary problem

$$
\begin{aligned}
\Delta P(y, \cdot)=0 & \text { in } \Omega \\
P(y, \cdot)=\delta_{y}(\cdot) & \text { on } \Gamma .
\end{aligned}
$$

Lions's formula reads now ([L2], (2.13)):

$$
K(x, b)=\int_{\Gamma} M_{y}^{-\frac{s}{2}} N_{y} G(y, x) \cdot M_{y}^{-\frac{s}{2}} N_{y} G(y, b) d \sigma(y)
$$

valid for any $x, b \in \Omega$. Here $K(x, b)$, or more fully $K_{s}(x, b)$ (indicating the dependence on $s$ ), is the reproducing kernel in $\mathscr{H}^{s}(\Omega)$.

Using the Poisson kernel (1.11) the formula can be written more compactly as

$$
K(x, b)=\int_{\Gamma} M_{y}^{-\frac{s}{2}} P(y, x) \cdot M_{y}^{-\frac{s}{2}} P(y, b) d \sigma(y)
$$

Remark 1.5. Note that in [L2] the Poisson kernel is not mentioned.

In the proof of (1.12) Lions used the variational description of the reproducing kernel (see (1.4)-(1.5)). Indeed, using penalization, which used to be one of Lions's favorite ideas, he considered, corresponding to (1.5), the modified minimum problem

$$
\inf \left(\|u\|_{\mathscr{H}^{s}(\Omega)}^{2}+\frac{1}{\varepsilon}\|\Delta u\|_{L^{2}(\Omega)}^{2}\right), \quad u(b)=1,
$$

5) As in [L2], it will be assumed throughout that our domain $\Omega$ is such that $\Omega$ lies on one side of $\Gamma$ only, so it is legitimate to speak of the inner and outer normal direction. 
where $\varepsilon>0$ is a parameter. For details, see [L2], Section 3, notably (3.5) and (3.6). Let $w_{\varepsilon}$ be the unique solution to (1.13). Using compactness he picked a weakly convergent subsequence and so, after some auxiliary partial integrations, he arrived at the desired result (1.12).

In the present paper we develop several alternative approaches to (1.12) including several more general similar formulae. Thereby we reduce Lions's formula to first principles, or so-called 'general nonsense'. However, it should be kept in mind that it is the discovery of a theorem or a formula that matters, afterwards everybody can streamline the proof. It is also not clear whether Lions himself would have approved our approach.

A major ingredient in one version of our proof is the observation of a relation between the reproducing kernel and the Poisson kernel. In the simplest case this is embodied in the formula

$$
\gamma K_{x}=M^{-s} P_{x}
$$

We observe that as $K_{x}$ is a harmonic function it is determined by its boundary values.

Proof of (1.12) via (1.14). Indeed, taking (1.14) as granted we obtain (see (1.2))

$$
K(x, b)=\left(K_{b}, K_{x}\right)_{\mathscr{H}^{s}(\Omega)}=\left(M^{-s} P_{b}, M^{-s} P_{x}\right)_{H^{s}(\Gamma)}=\left(M^{-s} P_{b}, P_{x}\right)_{L^{2}(\Gamma)},
$$

which apparently is the same as (1.12).

So in the light of this Lions's formula is just a sort of recasting of the fundamental identity (1.2)!

For the Reader's benefit, we indicate here also a simple deduction of (1.14).

Proof of (1.14). Let $f$ be any harmonic function in $\Omega$. Then by the reproducing property of the Poisson kernel we have for $x \in \Omega$

$$
f(x)=\int_{\Gamma} f(y) P_{x}(y) d \sigma(y)=\left(f, P_{x}\right)_{L^{2}(\Gamma)} .
$$

(Strictly speaking, we should write here $\gamma f(y)$ and $\left(\gamma f, P_{x}\right)_{L^{2}(\Gamma)}$, but to make the formulas simpler we will omit the $\gamma$.) If $f \in \mathscr{H}^{s}(\Omega)$ we can write the last inner product as

$$
\left(f, P_{x}\right)_{L^{2}(\Gamma)}=\left(M^{s} f, M^{-s} P_{x}\right)_{L^{2}(\Gamma)}=\left(f, M^{-s} P_{x}\right)_{H^{s}(\Gamma)},
$$

so that

$$
f(x)=\left(f, M^{-s} P_{x}\right)_{H^{s}(\Gamma)} .
$$

Again if we compare this equality with the definition of the reproducing kernel (see (1.1) and (1.9)), we see that (1.14) follows.

At the end of his paper [L2], Lions indicated several extensions of his result. For instance, in Remark 4.4 he said: "In all what has been said one can replace the condition 
[L2], (4.12) $\Delta u=0$

by

[L2], (4.13) $\quad A u=0$

where $A$ is any elliptic operator (with $C^{\infty}$ coefficients), of any order." Especially, he quotes the case of biharmonic, rather than harmonic functions. He says further that a similar remark applies if one replaces [L2], (4.12) by a hypoelliptic equation, such as the heat equation

$$
\frac{\partial u}{\partial t}-\Delta u=0 \quad \text { in } \Omega \times(0, T) .
$$

Similarly, in Remark 4.5 he makes some observations on the case of Neumann, rather than Dirichlet, boundary conditions. However, no details are given about any of this.

In Sections 2-4 of the present paper we develop several general approaches to reproducing formulae of the type of the Lions formula (1.12). In particular, we cover thereby some of the above cases left open by Lions [L2], which we have referred to above, and also (in Section 4) some classical formulae due to Garabedian [G1], Bergman-Schiffer [BS] and Zaremba $[\mathrm{Z} 1]$.

In Section 5 we give some concrete examples of reproducing kernels for special domains $\Omega$. These are all cases when the method of separation of variables (or Fourier's method) can be applied due to the symmetries of $\Omega$. Therefore an orthonormal basis can easily be found, so in view of (1.10) the reproducing kernel can be written down explicitly. Formally speaking, Lions's formula is not required here. Likewise, the whole section may be read right after this Introduction. On the other hand, on the basis of such explicit formulae the validity of Lions's formula can be checked in unbounded situations also (here we treat the case of the halfplane only). Note that all our examples are two dimensional. This is because of simplicity. But without doubt similar examples can be given in any number of dimensions. To have concrete expressions for the reproducing kernel may be of independent interest as has, in part, been dictated by the third author's fascination of special functions! While many explicit expressions for the reproducing kernel are known in the holomorphic case (see e.g. [B]), rather little of this sort seems to have been known in the harmonic case (cf. however the early paper [Z2]). Roughly speaking, we are in this way led to generalizations of known special functions depending on the smoothness parameter $s$, so we may refer to them as s-generalizations. In particular, in treating the heat equation we are led in Subsections 5.3-5.4, in the case of periodic solutions, to such $s$-generalizations of the theta functions both in the original Jacobi case of one variable as in the general case of $p$ variables. However, we do not know yet if these functions possess any properties equally interesting as those in the classical case.

Remark 1.6. One more reason why Lions's formula seems interesting is that these ways of formulating the problems seem to have much in common with other types of formulations, like integral equations of certain types, in the sense that they are "working" on the boundary rather than in the interior (see e.g. $[\mathrm{GH}]$ ). Such formulations have proven to give stable numerical schemes and enable computation of the actual solution with accuracy 
much better than e.g. the finite element method (which is based on a standard weak formulation of the underlying PDE).

We conclude this Introduction by making another remark indicating a connection between the original Lions formula, in the case of harmonic functions, and the classical variational formula of Hadamard for the Green's function. ${ }^{6)}$

Remark 1.7. Let the generic point $x \in \Omega$ move to the infinitely close point $x+\delta x$. Then, as Hadamard $[\mathrm{H}]$ showed, the quantity $G(x, b)$, where $x, b \in \Omega$, experiences a change given by

$$
\delta G(x, b)=\int_{\Gamma} P(y, x) P(y, b) \delta N(y) d \sigma(y)
$$

where $\delta N$ or, more fully, $\delta N(y), N=N(y)$ being the inner normal at the point $y \in \Gamma$, is defined by

$$
\delta N=(N(y), \delta y)
$$

the inner product being the Euclidean one in $\mathbb{R}^{n}$. The similarity between (1.12) and (1.15) is plain. Let us elaborate this a little bit more. To this end introduce the notation

$$
J(y, z):=P(y, x) P(z, b)
$$

for a moment we consider $x$ and $b$ as fixed, at least we need not indicate them in our notation. View $J$ as a "kernel". Thus if we consider the corresponding integral operator, say $\mathscr{J}$, we recognize therein $(1.15)$. So very symbolically we may rewrite this formula simply as

$$
\delta G=\operatorname{trace}(\mathscr{J} \cdot \delta N)
$$

where the last $\delta N$ is to be interpreted as the operator of multiplication by the function $\delta N(y) .^{7)}$

To recover also the Lions formula (1.12) we must first apply the operator $M_{y}^{-\frac{s}{2}} \otimes M_{z}^{-\frac{s}{2}}$. Let the so-modified kernel be written $J_{s}(y, z)$. Then the reproducing kernel $K_{s}(x, b)$ arises just as the trace of $J_{s}$ :

$$
K_{s}=\operatorname{trace}\left(J_{S}\right)
$$

We are, however, not aware of the deeper reasons for this phenomenon, if any.

Generalizations of Hadamard's formula to higher order elliptic boundary problems have been proposed by D. Fujiwara and S. Ozawa [FO] and by J. Peetre [Pe3] (although in the latter paper only special elliptic boundary problems coming from an energy integral are

6) Indeed, it was this analogy, although perhaps a false one, that originally caused the third author to undertake this research!

7) Note that the word trace is now used in a different, algebraic sense; hitherto we spoke just of the trace of a distribution on a submanifold, which is a quite different thing. 
considered in rather restricted assumptions). There arises the question whether the considerations in the previous paragraph can be extended to the higher order situation.

We have however serious doubts that this can be done. Thus we are of the opinion that the connection between Hadamard's formula and the reproducing kernel just indicated, in the classical harmonic case, seems to be an isolated phenomenon peculiar to the second order case.

\section{Lions's formula for determinate elliptic problems}

Let $\Omega$ be a bounded domain in $\mathbb{R}^{n}$ with smooth $\left(=C^{\infty}\right)$ boundary $\Gamma$, and $L^{2}(\Gamma)$ the usual Lebesgue space on $\Gamma$ with respect to the $(n-1)$-dimensional surface measure $\sigma$. Let further $\Delta_{\Gamma}$ be the Laplace-Beltrami operator on $\Gamma$, and set

$$
M=I-\Delta_{\Gamma}
$$

where $I$ is the identity operator on $L^{2}(\Gamma)$. It is then well known that $M^{-1}$ is a bounded selfadjoint nonnegative-definite operator on $L^{2}(\Gamma)$ and, hence, the powers $M^{s}$ make sense for any complex $s$.

For $s \in \mathbb{R}$, the Sobolev space $H^{s}(\Gamma)$ is the completion of $C^{\infty}(\Gamma)$ with respect to the norm induced by the inner product

$$
(u, v)_{H^{s}(\Gamma)}:=\left(M^{s} u, v\right)_{L^{2}(\Gamma)}
$$

For $s \geqq 0$, it is easily seen that $H^{s}(\Gamma) \subset H^{0}(\Gamma)=L^{2}(\Gamma)$, and, in fact, $H^{s}(\Gamma)$ coincides with the domain of the (unbounded) selfadjoint operator $M^{s / 2}$; hence, in particular, the elements of $H^{s}(\Gamma)$ can be identified with functions on $\Gamma$. For $s<0, H^{s}(\Gamma) \subset \mathscr{D}^{\prime}(\Gamma)$, the space of distributions on $\Gamma$, and in fact one has the equalities

$$
\bigcup_{s \in \mathbb{R}} H^{s}(\Gamma)=\mathscr{D}^{\prime}(\Gamma), \quad \bigcap_{s \in \mathbb{R}} H^{s}(\Gamma)=C^{\infty}(\Gamma)
$$

We further denote by $H^{s}(\Omega)$ the Sobolev spaces on the domain $\Omega$, by $\gamma$ the operator of trace on the boundary, and by $N:=\frac{\partial}{\partial n}$ the derivative in the direction of the exterior normal on $\Gamma$. Then $\gamma$ is a well-defined bounded operator from $H^{s}(\Omega)$ into $H^{s-\frac{1}{2}}(\Gamma)$, for any $s>1 / 2$, and so are, in fact, $\gamma N^{j}$ from $H^{s}(\Omega)$ into $H^{s-j-\frac{1}{2}}(\Gamma)$, for all nonnegative integers $j<s-(1 / 2)$. The intersection of the kernels of all $\gamma N^{j}, 0 \leqq j<s-(1 / 2)$, is precisely $H_{0}^{s}(\Omega)$, the closure of $\mathscr{D}(\Omega)$ in $H^{s}(\Omega)$. Unlike the compact situation for $\Gamma$, this time one has instead of (2.1) the equalities

$$
\bigcap_{s \in \mathbb{R}} H^{s}(\Omega)=C^{\infty}(\bar{\Omega}),
$$

by the Sobolev lemma, and

$$
\bigcup_{s \in \mathbb{R}} H^{s}(\Omega)=: \mathscr{D}^{\prime}(\bar{\Omega}) \varsubsetneqq \mathscr{D}^{\prime}(\Omega),
$$


the subspace of distributions on $\Omega$ that can be continued as distributions to a neighbourhood of $\bar{\Omega}$.

Let now $L$ be a linear partial differential operator of order $2 m$ on $\Omega$ with coefficients in $C^{\infty}(\bar{\Omega})$, and $B_{j}(j=0, \ldots, m-1)$ an $m$-tuple of differential "boundary operators" of orders $m_{j}$ on $\Gamma$ whose coefficients are $C^{\infty}$ in some neighbourhood of $\Gamma$. We will assume that the boundary value problem

$$
\begin{aligned}
L u & =0 \quad \text { on } \Omega, \\
\gamma B_{j} u & =f_{j} \quad \text { on } \Gamma, \quad \forall j=0,1, \ldots, m-1,
\end{aligned}
$$

is elliptic, i.e. that $L$ is a properly elliptic operator on $\bar{\Omega}, B_{j}$ form a normal system of operators on $\Gamma$, their orders $m_{j}$ are pairwise distinct and $\leqq 2 m-1$, and $B_{j}$ cover $L$ on $\Gamma$ (see [LM], Chapter 2, §5.1, or [Fo], Section 7.G). (Here the "boundary trace" $\gamma$ has to be interpreted suitably if $u \notin C^{\infty}(\bar{\Omega})$, see e.g. [LM], Chapter 2, §8.1.)

Let $L^{+}$be the formal adjoint of $L$ and $S_{j}(j=0, \ldots, m-1)$ be any normal $m$-tuple of boundary operators on $\Gamma$ whose coefficients are $C^{\infty}$ in some neighbourhood of $\Gamma$ and whose orders $c_{j}$ are such that $\left\{m_{0}, \ldots, m_{m-1}\right\} \cup\left\{c_{0}, \ldots, c_{m-1}\right\}=\{0, \ldots, 2 m-1\}$. Then there exist uniquely determined operators $B_{j}^{+}$and $S_{j}^{+}(j=0, \ldots, m-1)$, of orders $2 m-1-c_{j}$ and $2 m-1-m_{j}$, respectively, such that the following Green formula

$$
\int_{\Omega}\left(L u \cdot v-u \cdot L^{+} v\right) d \tau=\sum_{j=0}^{m-1} \int_{\Gamma}\left(\gamma S_{j} u \cdot \gamma B_{j}^{+} v-\gamma B_{j} u \cdot \gamma S_{j}^{+} v\right) d \sigma
$$

holds for all $u, v \in C^{\infty}(\bar{\Omega})$. (See $[\mathrm{LM}]$, Chapter 2, §2.2.)

Here, and in the sequel, $\tau$ stands for the Lebesgue measure on $\mathbb{R}^{n}$.

It is known that under the above ellipticity hypotheses, the problem (2.2) has a solution for any $f_{0}, \ldots, f_{m-1} \in \mathscr{D}^{\prime}(\Gamma)$ satisfying

$$
\sum_{j=0}^{m-1} \int_{\Gamma} f_{j} \cdot \gamma S_{j}^{+} v d \sigma=0 \quad \forall v \in \mathscr{N}^{+}
$$

where

$$
\mathscr{N}^{+}:=\left\{v \in C^{\infty}(\bar{\Omega}): L^{+} v=0 \text { on } \Omega, \gamma B_{j}^{+} v=0 \text { on } \Gamma \forall j\right\}
$$

and the solution $u$ belongs to $\mathscr{D}^{\prime}(\bar{\Omega}) \cap C^{\infty}(\Omega)$ and is unique up to a summand from

$$
\mathscr{N}:=\left\{u \in C^{\infty}(\bar{\Omega}): L u=0 \text { on } \Omega, \gamma B_{j} u=0 \text { on } \Gamma \forall j\right\}
$$

(See e.g. [LM], Chapter 2, §7.3.)

From now on until the end of this section we will assume that the problem (2.2) is determinate, i.e. that 


$$
\mathscr{N}=\mathscr{N}^{+}=\{0\} .
$$

Then the solutions to (2.2) can be obtained by using the above Green formula, as follows. For each $a \in \Omega$, the adjoint problem

$$
\begin{aligned}
& L^{+} v=\delta_{a} \quad \text { on } \Omega(\text { the point mass at } a), \\
& B_{j}^{+} v=0 \quad \text { on } \Gamma \quad \forall j=0, \ldots, m-1,
\end{aligned}
$$

is also determinate elliptic and thus has a unique solution

$$
v=: G_{a} \in H^{2 m-\frac{n}{2}}(\Omega) \cap C^{\infty}(\bar{\Omega} \backslash\{a\}) ;
$$

$G(x, a):=G_{a}(x)$ is called the Green function of (2.2). Applying the formula (2.3) with $v=G_{a}$ and $u$ as in (2.2), we see that the solution to (2.2) (which is unique by (2.6)) is given by

$$
u(a)=\sum_{j=0}^{m-1} \int_{\Gamma} f_{j} \cdot P_{a}^{(j)} d \sigma
$$

where the Poisson kernels $P_{a}^{(j)}$ are given by

$$
P_{a}^{(j)}:=\gamma S_{j}^{+} G_{a} \in C^{\infty}(\Gamma)
$$

The corresponding Poisson operators

$$
\mathbf{P}^{(j)} f(a):=\int_{\Gamma} f \cdot P_{a}^{(j)} d \sigma
$$

are known to be continuous from $H^{s-m_{j}-1 / 2}(\Gamma)$ into $H^{s}(\Omega)$, for each $s \in \mathbb{R}$. (See again e.g. $[\mathrm{LM}]$, Chapter 2, §7.3.) We will also employ the notation $\mathbf{P}$ for the collective Poisson operator

$$
\mathbf{P}: \quad \mathbf{f}:=\left(f_{0}, \ldots, f_{m-1}\right) \mapsto \sum_{j=0}^{m-1} \mathbf{P}^{(j)} f_{j}=: \mathbf{P f}
$$

so that (2.8) can be written as $u=\mathbf{P f}$.

Now define, for any $\boldsymbol{s}=\left(s_{0}, \ldots, s_{m-1}\right) \in \mathbb{R}^{m}$,

$$
\begin{aligned}
& H^{s}(\Gamma):=H^{s_{0}}(\Gamma) \oplus \cdots \oplus H^{s_{m-1}}(\Gamma) \\
& \mathscr{H}^{s}(\Omega):=\left\{u \in \mathscr{D}^{\prime}(\bar{\Omega}): L u=0, \gamma B_{j} u \in H^{s_{j}}(\Gamma) \forall j=0, \ldots, m-1\right\}=\mathbf{P} H^{s}(\Gamma),
\end{aligned}
$$

endowed with the scalar product

$$
(u, v)_{\mathscr{H}^{s}(\Omega)}:=\sum_{j=0}^{m-1}\left(\gamma B_{j} u, \gamma B_{j} v\right)_{H^{s_{j}}(\Gamma)} .
$$

Having collected these preliminaries, we can now state the main result of this section. 
given by

Theorem 2.1. The space $\mathscr{H}^{s}(\Omega)$ possesses a reproducing kernel $K_{y}(x) \equiv K(x, y)$

$$
K(x, y)=\sum_{j=0}^{m-1} \int_{\Gamma} M^{-s_{j} / 2} P_{y}^{(j)} \cdot M^{-s_{j} / 2} P_{x}^{(j)} d \sigma .
$$

Proof. Let $\mathbf{f} \in H^{s}(\Gamma)$. By (2.8), for any $y \in \Omega$

$$
\begin{aligned}
\mathbf{P f}(y) & =\sum_{j=0}^{m-1}\left(f_{j}, P_{y}^{(j)}\right)_{L^{2}(\Gamma)} \\
& =\sum_{j=0}^{m-1}\left(M^{s_{j}} f_{j}, M^{-s_{j}} P_{y}^{(j)}\right)_{L^{2}(\Gamma)} \\
& =\sum_{j=0}^{m-1}\left(f_{j}, M^{-s_{j}} P_{y}^{(j)}\right)_{H^{s_{j}}(\Gamma)} .
\end{aligned}
$$

(Note that $M^{-s} P_{y}^{(j)}$ is well defined and belongs to $H^{s}(\Gamma)$, for any $s$, since $P_{y}^{(j)} \in C^{\infty}(\Gamma)$.) On the other hand, $K_{y}$ is the element of $\mathscr{H}^{s}(\Omega)$ uniquely determined by the reproducing property

$$
\mathbf{P f}(y)=\left(\mathbf{P f}, K_{y}\right)_{\mathscr{H}^{s}(\Omega)}=\sum_{j=0}^{m-1}\left(f_{j}, \gamma B_{j} K_{y}\right)_{H^{s j}(\Gamma)} \quad \forall \mathbf{f} \in H^{s}(\Gamma)
$$

Thus we must have

$$
\begin{array}{rlrl}
L K_{y} & =0 & & \text { on } \Omega, \\
\gamma B_{j} K_{y} & =M^{-s_{j}} P_{y}^{(j)} & \forall j,
\end{array}
$$

whence by $(2.8)$

$$
\begin{aligned}
K_{y}(x) & =\mathbf{P}\left(M^{-s_{0}} P_{y}^{(0)}, \ldots, M^{-s_{m-1}} P_{y}^{(m-1)}\right)(x) \\
& =\sum_{j=0}^{m-1}\left(M^{-s_{j}} P_{y}^{(j)}, P_{x}^{(j)}\right)_{L^{2}(\Gamma)} \\
& =\sum_{j=0}^{m-1} \int_{\Gamma} M^{-s_{j}} P_{y}^{(j)} \cdot P_{x}^{(j)} d \sigma
\end{aligned}
$$

which is plainly equivalent to $(2.11)$. theorem,

Corollary 2.2 (Generalized Lions's formula). Under the hypothesis of the preceding

$$
K(x, y)=\sum_{j=0}^{m-1} \int_{\Gamma} M^{-s_{j} / 2} S_{j}^{+} G_{y} \cdot M^{-s_{j} / 2} S_{j}^{+} G_{x} d \sigma .
$$


Proof. Immediate from (2.11) and (2.9).

Example 2.3. Let $L=\Delta$, the Laplace operator on $\Omega$, and $B_{0}=I$, the identity operator, so that $G_{y}(x) \equiv G(x, y)$ is the ordinary Green function for the Laplacian with the Dirichlet boundary conditions. Then $S_{0}^{+}=N$, the (exterior) normal derivative, and (2.12) becomes

$$
K(x, y)=\int_{\Gamma} M^{-s / 2} N G_{y} \cdot M^{-s / 2} N G_{x} d \sigma
$$

which is the original Lions formula from [L2].

Example 2.4. Taking next the biharmonic operator $L=\Delta^{2}$, with the Dirichlet boundary conditions $B_{0}=I, B_{1}=N$, so that $G$ is the biharmonic Green function which we denote by $B(x, y) \equiv B_{y}(x)$. The operators $S_{j}^{+}$are then given by $S_{0}^{+}=N \Delta, S_{1}^{+}=-\Delta$ (cf. e.g. $[\mathrm{EP}])$. Thus (2.12) yields

$$
K(x, y)=\int_{\Gamma}\left(M^{-s_{0} / 2} \gamma N \Delta B_{y} \cdot M^{-s_{0} / 2} \gamma N \Delta B_{x}+M^{-s_{1} / 2} \gamma \Delta B_{y} \cdot M^{-s_{1} / 2} \gamma \Delta B_{x}\right) d \sigma
$$

which is the simplest higher-order generalization of Lions's formula.

\section{The indeterminate case}

In principle, one can consider the spaces $\mathscr{H}^{s}(\Omega)$ also if $\mathscr{N} \neq\{0\}$; however, in that case the "scalar product" (2.10) is no longer positive definite, but only positive semidefinite (the elements of $\mathscr{N}$ have zero norm), and, consequently, the evaluation functionals $u \mapsto u(x)$ are no longer continuous with respect to it (if $0 \neq u \in \mathscr{N}$, then $\|u\|=0$ but $u(x) \neq 0$ for some $x$ ). Thus there exists no reproducing kernel. What one has to do is either to pass from $\mathscr{H}^{s}(\Omega)$ to a suitable subspace which is complementary to $\mathscr{N}$, or to modify the inner product $(2.10)$ by adding to it an extra term $\left(\right.$ like $\left.\|u\|_{L^{2}(\Omega)}\right)$ which makes it positive definite.

Here is an example (of the former approach). Let $N$ stand, as before, for the exterior normal derivative, and let further $F(x, y) \equiv F_{y}(x)$ be the Neumann function for the Laplace operator on $\Omega$, i.e. the solution of the boundary value problem

$$
\begin{aligned}
\Delta F_{a} & =\delta_{a} \quad(\text { the point mass at } a), \\
\gamma N F_{a} & =-\frac{1}{\sigma(\Gamma)} \quad \text { on } \Gamma, \\
\int_{\Gamma} \gamma F_{a} d \sigma & =0 .
\end{aligned}
$$

By the general theory recalled at the beginning of the preceding section, the Neumann problem for $\Delta$, 


$$
\begin{aligned}
\Delta u & =0, \\
\gamma N u & =f, \\
\int_{\Gamma} \gamma u d \sigma & =0
\end{aligned}
$$

is solvable if and only if

$$
\int_{\Gamma} f d \sigma=0
$$

and its unique solution is then given by

$$
u(y)=-\int_{\Gamma} f \cdot \gamma F_{y} d \sigma .
$$

Theorem 3.1. The space

$$
\mathscr{H}_{\text {Neum }}^{s}(\Omega):=\left\{u \in \mathscr{D}^{\prime}(\bar{\Omega}): \Delta u=0, \gamma N u \in H^{s-1}(\Gamma), \int_{\Gamma} \gamma u d \sigma=0\right\}
$$

with the scalar product

$$
(u, v)_{\mathscr{H}_{\mathrm{Neum}}^{s}(\Omega)}:=(\gamma N u, \gamma N v)_{H^{s-1}(\Gamma)}
$$

has the reproducing kernel

$$
K(x, y)=\int_{\Gamma} M^{(1-s) / 2} \gamma F_{y} \cdot M^{(1-s) / 2} \gamma F_{x} d \sigma .
$$

Proof. By (3.4), we have for any $u \in \mathscr{H}_{\text {Neum }}^{s}(\Omega)$ and $y \in \Omega$,

$$
u(y)=\left(\gamma N u,-\gamma F_{y}\right)_{L^{2}(\Gamma)} .
$$

On the other hand, the reproducing kernel $K(x, y) \equiv K_{y}(x)$ is the uniquely determined element of $\mathscr{H}_{\text {Neum }}^{s}(\Omega)$ satisfying

$$
u(y)=\left(\gamma N u, \gamma N K_{y}\right)_{H^{s-1}(\Gamma)}=\left(\gamma N u, M^{s-1} \gamma N K_{y}\right)_{L^{2}(\Gamma)}
$$

for all $u \in \mathscr{H}_{\mathrm{Neum}}^{s}(\Omega)$. Thus

$$
\left(f,-\gamma F_{y}\right)_{L^{2}(\Gamma)}=\left(f, M^{s-1} \gamma N K_{y}\right)_{L^{2}(\Gamma)}
$$

whenever $f=\gamma N u$ for some $u$, i.e. for all functions $f$ satisfying (3.3). As

$$
\left(1, \gamma F_{y}\right)_{L^{2}(\Gamma)}=0
$$

(by the third condition in (3.1)) and

$$
\left(1, M^{s-1} \gamma N K_{y}\right)_{L^{2}(\Gamma)}=\left(M^{s-1} 1, \gamma N K_{y}\right)_{L^{2}(\Gamma)}=\left(1, \gamma N K_{y}\right)_{L^{2}(\Gamma)}=0
$$


(by the fact that $M 1=1$ and (3.3)), we see that (3.5) actually holds for all $f$, so

$$
\gamma N K_{y}=-M^{1-s} \gamma F_{y}
$$

Thus by (3.4)

$$
K_{y}(x)=-\int_{\Gamma} \gamma N K_{y} \cdot \gamma F_{x} d \sigma=\int_{\Gamma} M^{1-s} \gamma F_{y} \cdot \gamma F_{x} d \sigma
$$

which is the desired assertion.

The rest of this section is devoted to showing that a similar result can be established for the general elliptic problem (2.2), as long as the Cauchy problem for $L$ is uniquely solvable and the subspaces $\gamma S_{j}^{+} \mathscr{N}^{+}, j=0, \ldots, m-1$, are invariant under the operator $M$ (or, equivalently, $\Delta_{\Gamma}$ ). (In the last proof, the latter corresponds to the equality $M 1=1$.) This, unfortunately, clearly need not be the case in general.

In order to state the result properly, we need first to introduce some notation and to prove a proposition.

Let $L, B_{j}, S_{j}, \mathscr{N}$, etc., be as in the beginning of Section 2. Until the end of this section, we will assume that the Cauchy problems for $L$ and for its adjoint $L^{+}$are uniquely solvable, i.e.

$$
\left(L u=0 \text { on } \Omega, \gamma B_{j} u=\gamma S_{j} u=0 \text { on } \Gamma \forall j=0, \ldots, m-1\right) \Rightarrow u \equiv 0,
$$

and similarly for $L^{+}$. (This is, for instance, always the case when $L$ has real-analytic coefficients, by Holmgren's theorem.)

Consider now the following general analogue of the Neumann problem (3.2):

$$
\begin{aligned}
L u & =0, \\
\gamma B_{j} u & =f_{j}, \quad \forall j=0, \ldots, m-1, \\
\int_{\Gamma} \gamma S_{j} u \cdot \gamma S_{j} w d \sigma & =0 \quad \forall w \in \mathscr{N} .
\end{aligned}
$$

Introducing the vector notation

$$
\boldsymbol{B} u:=\left(B_{0} u, \ldots, B_{m-1} u\right), \quad \boldsymbol{S} u:=\left(S_{0} u, \ldots, S_{m-1} u\right),
$$

this can be rewritten more succinctly as

$$
\begin{gathered}
L u=0, \\
\gamma \boldsymbol{B} u=\mathbf{f}, \\
\gamma \boldsymbol{S} u \perp \gamma \boldsymbol{S} \mathscr{N} .
\end{gathered}
$$


(The symbol $\perp$ denotes orthogonality in the Cartesian product $L^{2}(\Gamma)^{m}$ of $m$ copies of $L^{2}(\Gamma)$.) In view of the assumption (3.6) and the general theory recalled at the beginning of the preceding section, this problem is solvable if and only if

$$
\mathbf{f} \perp \gamma \boldsymbol{S}^{+} v \quad \forall v \in \mathscr{N}^{+}
$$

and then the third condition in (3.7) determines the solution uniquely.

Similarly, the adjoint elliptic problem

$$
L^{+} v=\phi, \quad \gamma \boldsymbol{B}^{+} v=\mathbf{g}, \quad \gamma \boldsymbol{S}^{+} v \perp \gamma \boldsymbol{S}^{+} \mathscr{N}^{+}
$$

is solvable if and only if

$$
(u, \phi)_{L^{2}(\Omega)}=-(\gamma \boldsymbol{S} u, \mathbf{g})_{L^{2}(\Gamma)^{m}} \quad \forall u \in \mathscr{N}
$$

and then the solution is unique.

Finally, we introduce the following scalar product on the space $\mathscr{N}$ :

$$
(u, v)_{\mathscr{N}}:=(\gamma \boldsymbol{S} u, \gamma \boldsymbol{S} v)_{L^{2}(\Gamma)^{m}}
$$

(It follows from (3.6) that this is indeed a scalar product, i.e. $\|u\|_{\mathscr{N}}=0$ only for $u \equiv 0$.) As $\mathscr{N}$ is finite dimensional, the evaluation functionals on it are automatically continuous with respect to $(3.10)$, and thus $\mathscr{N}$ possesses a reproducing kernel which we denote by $\kappa(x, y) \equiv \kappa_{y}(x)$.

Proposition 3.2. For $a \in \Omega$, let $v$ be the (unique) solution to the adjoint problem

$$
\begin{gathered}
L^{+} v=\delta_{a}, \\
\gamma \boldsymbol{B}^{+} v=-\gamma \boldsymbol{S} \kappa_{a}, \\
\gamma \boldsymbol{S}^{+} v \perp \gamma \boldsymbol{S}^{+} \mathscr{N}^{+},
\end{gathered}
$$

and set

$$
\boldsymbol{Q}_{a}:=\gamma \boldsymbol{S}^{+} v \in L^{2}(\Gamma)^{m}
$$

Then the solution to the problem (3.7) is given by

$$
u(a)=\left(\mathbf{f}, \boldsymbol{Q}_{a}\right)_{L^{2}(\Gamma)^{m}} \quad \forall a \in \Omega
$$

Proof. By the reproducing property of $\kappa_{a}$, we have

$$
u(a)=\left(\gamma \boldsymbol{S} u, \gamma \boldsymbol{S} \kappa_{a}\right)_{L^{2}(\Gamma)^{m}} \quad \forall u \in \mathscr{N}
$$

Thus it follows from (3.9) that (3.11) is indeed solvable. Denoting by $v$ its unique solution and letting $u$ be a solution to (3.7), the Green formula (2.3) gives 


$$
\begin{aligned}
u(a) & =\int_{\Omega}\left(u \cdot L^{+} v-L u \cdot v\right) d \tau \\
& =\left(\gamma \boldsymbol{B} u, \gamma \boldsymbol{S}^{+} v\right)_{L^{2}(\Gamma)^{m}}-\left(\gamma \boldsymbol{S} u, \gamma \boldsymbol{B}^{+} v\right)_{L^{2}(\Gamma)^{m}} \\
& =\left(\mathbf{f}, \gamma \boldsymbol{S}^{+} v\right)_{L^{2}(\Gamma)^{m}}+\left(\gamma \boldsymbol{S} u, \gamma \boldsymbol{S} \kappa_{a}\right)_{L^{2}(\Gamma)^{m}}
\end{aligned}
$$

Since $\kappa_{a} \in \mathscr{N}$, the second summand vanishes by the third condition in (3.7), and the assertion follows.

Now we are ready to state the promised theorem.

Theorem 3.3. Assume that for each $0 \leqq j \leqq m-1$, the operator $M$ maps the space $\gamma S_{j} \mathscr{N}^{+} \subset L^{2}(\Gamma)$ into itself:

$$
M \gamma S_{j} \mathscr{N}^{+} \subset \gamma S_{j} \mathscr{N}^{+} \quad \forall j=0, \ldots, m-1 .
$$

Then the reproducing kernel for the space

$$
\mathscr{H}^{s}(\Omega):=\left\{u \in \mathscr{D}^{\prime}(\bar{\Omega}): L u=0, \gamma \boldsymbol{B} u \in H^{s}(\Gamma), \gamma \boldsymbol{S} u \perp \gamma \boldsymbol{S} \mathscr{N}\right\}
$$

with the scalar product

$$
(u, v)_{\mathscr{H}^{s}(\Omega)}:=\sum_{j=0}^{m-1}\left(\gamma B_{j} u, \gamma B_{j} v\right)_{H^{s_{j}}(\Gamma)}
$$

is given by

$$
K(x, y)=\sum_{j=0}^{m-1} \int_{\Gamma} M^{-s_{j}} Q_{y}^{(j)} \cdot Q_{x}^{(j)} d \sigma
$$

where $\boldsymbol{Q}_{a}:=\left(Q_{a}^{(0)}, \ldots, Q_{a}^{(m-1)}\right)$.

Proof. Introducing, for brevity, the operator

$$
M^{s}:\left(f_{0}, \ldots, f_{m-1}\right) \mapsto\left(M^{s_{0}} f_{0}, \ldots, M^{s_{m-1}} f_{m-1}\right)
$$

on $L^{2}(\Gamma)^{m},(3.13)$ becomes

$$
(u, v)_{\mathscr{H}^{s}(\Omega)}:=\left(M^{s} \gamma \boldsymbol{B} u, \gamma \boldsymbol{B} v\right)_{L^{2}(\Gamma)^{m}} .
$$

Let $a \in \Omega$. By the preceding proposition, for any $u \in \mathscr{H}^{s}(\Omega)$

$$
u(a)=\left(\gamma \boldsymbol{B} u, \boldsymbol{Q}_{a}\right)_{L^{2}(\Gamma)^{m}}
$$

On the other hand, the sought reproducing kernel $K_{a} \equiv K(\cdot, a)$ is uniquely determined by the two requirements that it belong to $\mathscr{H}^{s}(\Omega)$ and that it have the reproducing property

$$
u(a)=\left(\gamma \boldsymbol{B} u, M^{s} \gamma \boldsymbol{B} K_{a}\right)_{L^{2}(\Gamma)^{m}}
$$


that is, by (3.14),

$$
\boldsymbol{Q}_{a}-M^{s} \gamma \boldsymbol{B} K_{a} \perp \gamma \boldsymbol{B} u \quad \forall u \in \mathscr{H}^{s}(\Omega) .
$$

By (3.8), the last condition is equivalent to

$$
\boldsymbol{Q}_{a}-M^{s} \gamma \boldsymbol{B} K_{a} \in \gamma \boldsymbol{S}^{+} \mathscr{N}^{+}
$$

that is,

$$
M^{-s} \boldsymbol{Q}_{a}-\gamma \boldsymbol{B} K_{a} \in M^{-s} \gamma \boldsymbol{S}^{+} \mathscr{N}^{+}
$$

Now the hypothesis (3.12), in conjunction with the fact that $M$ is invertible, implies that $M^{-s} \gamma \boldsymbol{S}^{+} \mathscr{N}^{+}=\gamma \boldsymbol{S}^{+} \mathcal{N}^{+}$. Thus the last condition reads

$$
M^{-s} \boldsymbol{Q}_{a}-\gamma \boldsymbol{B} K_{a} \in \gamma \boldsymbol{S}^{+} \mathscr{N}^{+} .
$$

As, by (3.8) again, $\gamma \boldsymbol{B} K_{a} \perp \gamma \boldsymbol{S}^{+} \mathscr{N}^{+}$, we thus see that

$$
\gamma \boldsymbol{B} K_{a}=\pi_{1} M^{-s} \boldsymbol{Q}_{a}
$$

where $\pi_{1}$ denotes the orthogonal projection in $L^{2}(\Gamma)^{m}$ onto $\left(\gamma \boldsymbol{S}^{+} \mathscr{N}^{+}\right)^{\perp}$. Finally,

$$
\begin{aligned}
K(x, y) \equiv K_{y}(x) & =\left(\gamma \boldsymbol{B} K_{y}, \boldsymbol{Q}_{x}\right)_{L^{2}(\Gamma)^{m}} \quad \text { by Proposition } 3.2 \\
& =\left(\pi_{1} M^{-s} \boldsymbol{Q}_{y}, \boldsymbol{Q}_{x}\right)_{L^{2}(\Gamma)^{m}} \\
& =\left(M^{-s} \boldsymbol{Q}_{y}, \pi_{1} \boldsymbol{Q}_{x}\right)_{L^{2}(\Gamma)^{m}} \\
& =\left(M^{-s} \boldsymbol{Q}_{y}, \boldsymbol{Q}_{x}\right)_{L^{2}(\Gamma)^{m}},
\end{aligned}
$$

since $\pi_{1} \boldsymbol{Q}_{x}=\boldsymbol{Q}_{x}$ in view of the third condition in (3.11). This completes the proof.

Remark 3.4. Without the hypothesis (3.12), we get from (3.15) only the equation

$$
\begin{aligned}
\gamma \boldsymbol{B} K_{a}= & \text { the (non-orthogonal) projection of } M^{-s} \boldsymbol{Q}_{a} \text { onto the subspace } \\
& \left(\gamma \boldsymbol{S}^{+} \mathscr{N}^{+}\right)^{\perp} \text { along the subspace } M^{-s} \gamma \boldsymbol{S}^{+} \mathscr{N}^{+},
\end{aligned}
$$

which is much more involved than (3.16). We have not been able to obtain an analogue of Theorem 3.3 in this more general setting.

An example when the hypotheses of Theorem 3.3 are satisfied is when $\Omega$ is the unit disc $\{z:|z|<1\}$ in the complex plane $\mathbb{C} \simeq \mathbb{R}^{2}$ and the operators $L$ and $B_{j}$, $j=0,1, \ldots, m-1$, are rotation-invariant; that is, if we denote $f_{\theta}(z):=f\left(e^{i \theta} z\right)$, then $L\left(f_{\theta}\right)=(L f)_{\theta} \forall \theta \in \mathbb{R}$, and similarly for $B_{j}$. Indeed, in that case all the operators $S_{j}, B_{j}^{+}$and $S_{j}^{+}$are also rotation-invariant, and so is the space $\mathscr{N}^{+}$(i.e. $f_{\theta} \in \mathscr{N}^{+}$whenever $f \in \mathscr{N}^{+}$), and, hence, also the spaces $\gamma S_{j} \mathcal{N}^{+}$. As the latter are finite-dimensional, it follows easily that each of them must be of the form 


$$
\operatorname{span}\left\{z^{k}: k \in \mathfrak{\Xi}_{j}\right\}
$$

for some finite subsets $\mathfrak{S}_{j}$ of the integers. But $M z^{k}=\left(k^{2}+1\right) z^{k}$ for any integer $k$, and the assertion follows.

\section{An alternative approach}

In this section we describe another approach to Theorem 2.1 (for instance), which exploits the idea of deriving the result for a general $s$ from the corresponding result for the particular value $\boldsymbol{s}=(0, \ldots, 0)$. This approach also gives interesting insights into some (known) classical formulas for various reproducing kernels due to Bergman-Schiffer, Garabedian, and Zaremba. On the other hand, however, it does not seem powerful enough to yield the results on the indeterminate case (Theorem 3.3) from the preceding section (as one of the authors originally hoped).

The idea is to examine how a reproducing kernel is affected by a change of the scalar product.

Let us thus consider, quite generally, a reproducing kernel Hilbert space $\mathscr{E}$, with a scalar product $(,)_{\mathscr{E}}$ and reproducing kernel $K(x, y) \equiv K_{y}(x)$. Let $\mathscr{F}$ be another (quite arbitrary) Hilbert space, with scalar product $(,)_{\mathscr{F}}(\mathscr{F}$ is not assumed to have a reproducing kernel, in fact it need not be a space of functions at all). Finally, let $A$ be a densely defined and closed linear operator (possibly unbounded) from $\mathscr{E}$ into $\mathscr{F}$.

Let us now introduce a new inner product in $\mathscr{E}$ using the operator $A$. First of all, set

$$
[f, g]:=(A f, A g)_{\mathscr{F}} .
$$

In general, this is far from being an inner product: first, it is not defined for all $f, g \in \mathscr{E}$, but only for $f, g \in \operatorname{dom} A$; second, it may be only semi-definite; and third, it need not define a complete metric. We remedy this using the standard procedure: namely, denote

$$
\mathscr{M}_{A}:=\operatorname{dom} A \ominus \operatorname{ker} A \text {. }
$$

Then (4.1) is defined on $\mathscr{M}_{A}$ and is positive definite there (i.e. $[f, f]>0 \forall f \neq 0$ ). Let $\mathscr{E}_{A}$ be the completion of $\mathscr{M}_{A}$ with respect to the norm $\|f\|_{A}:=[f, f]^{1 / 2}$. Then $\mathscr{E}_{A}$ is a Hilbert space, with $\mathscr{M}_{A}$ as a dense subspace, and (4.1) extends to a well defined scalar product on $\mathscr{E}_{A}$.

Let us now investigate under what circumstances can the elements of the completion $\mathscr{E}_{A}$ be still viewed as functions; and if they can, whether the evaluation functionals are still continuous; and if they are, what is the relationship between the reproducing kernel $K(x, y)$ of $\mathscr{E}$ and the reproducing kernel of $\mathscr{E}_{A}$.

For the evaluation functional at some point $x$ to be continuous on $\mathscr{E}_{A}$, it is necessary and sufficient that $|f(x)| \leqq C[f, f]^{1 / 2}$ for all $f$ in the dense subset $\mathscr{M}_{A}$. The latter is equivalent to 


$$
\left|\left(f, K_{x}\right)_{\mathscr{E}}\right| \leqq C\|A f\|_{\mathscr{F}} \quad \forall f \in \mathscr{M}_{A},
$$

that is,

$$
\left|\left(f, \pi K_{x}\right)_{\mathscr{E}}\right| \leqq C\|A f\|_{\mathscr{F}} \quad \forall f \in \operatorname{dom} A
$$

where

$$
\pi: \mathscr{E} \rightarrow(\operatorname{ker} A)^{\perp}
$$

is the orthogonal projection in $\mathscr{E}$ onto $(\operatorname{ker} A)^{\perp}$. Let

$$
A=\left[\begin{array}{ll}
Y & 0 \\
0 & 0
\end{array}\right]:\left[\begin{array}{c}
(\operatorname{ker} A)^{\perp} \\
\operatorname{ker} A
\end{array}\right] \rightarrow\left[\begin{array}{c}
\overline{\operatorname{Ran} A} \\
(\operatorname{Ran} A)^{\perp}
\end{array}\right]
$$

be the matrix of $A$ with respect to the splittings $\mathscr{E}=(\operatorname{ker} A)^{\perp} \oplus \operatorname{ker} A$ and $\mathscr{F}=\overline{\operatorname{Ran} A} \oplus(\operatorname{Ran} A)^{\perp}=\left(\operatorname{ker} A^{*}\right)^{\perp} \oplus \operatorname{ker} A^{*}$ of $\mathscr{E}$ and $\mathscr{F}$, respectively; thus $Y$ is densely defined, closed, one-to-one, and with dense range, hence possesses an inverse $Y^{-1}$ with the same properties ${ }^{8}$. We can then continue the above chain of equivalences with

$$
\left|\left(Y^{-1} g, \pi K_{x}\right)_{\mathscr{E}}\right| \leqq C\|g\|_{\mathscr{F}} \quad \forall g \in \operatorname{Ran} A,
$$

which means precisely that

$$
\pi K_{x} \in \operatorname{dom}\left(Y^{-1}\right)^{*}=\operatorname{Ran} Y^{*}=\operatorname{Ran} A^{*}
$$

Thus if (4.2) is satisfied for all $x$, then the point evaluations extend continuously to all of $\mathscr{E}_{A}$, and, in particular, the elements of $\mathscr{E}_{A}$ can still be viewed as functions; and, further, $\mathscr{E}_{A}$ admits a reproducing kernel, say $L(x, y) \equiv L_{y}(x)$. From the considerations above we also see that

$$
f(x)=\left(f, K_{x}\right)_{\mathscr{E}}=\left(A f,\left(Y^{-1}\right)^{*} \pi K_{x}\right)_{\mathscr{F}}=\left(A f, A Y^{-1}\left(Y^{-1}\right)^{*} \pi K_{x}\right)_{\mathscr{F}},
$$

for every $f \in \mathscr{M}_{A}$, and it follows that

$$
L_{x}=Y^{-1}\left(Y^{-1}\right)^{*} \pi K_{x}=\left(Y^{*} Y\right)^{-1} \pi K_{x}
$$

(more precisely - this holds if $\pi K_{x}$ belongs to $\operatorname{dom}\left(Y^{*} Y\right)^{-1}=\operatorname{Ran} A^{*} A \subset \operatorname{Ran} A^{*}$; if $\pi K_{x} \in \operatorname{Ran} A^{*} \backslash \operatorname{Ran} A^{*} A$, then $L_{x}$ belongs to $\mathscr{E}_{A} \backslash \mathscr{M}_{A}$ and thus cannot be expressed directly).

For convenience, we summarize our findings in the following theorem.

Theorem 4.1. The reproducing kernel $L(x, y)$ of the space $\mathscr{E}_{A}$ exists if and only if $\pi K_{x} \in \operatorname{Ran} A^{*} \forall x \in \Omega$, and is then given by

8) In more detail: $Y$ is a densely defined, closed, and one-to-one operator with dense range from the closed subspace $\mathscr{E} \ominus \operatorname{ker} A$ of $\mathscr{E}$ into the closed subspace $\overline{\operatorname{Ran} A}$ of $\mathscr{F}$ (both subspaces being considered as Hilbert spaces of their own); similarly for $Y^{-1}$, with the roles of the two subspaces interchanged; and $\operatorname{dom} Y=\mathscr{M}_{A}=\operatorname{Ran} Y^{-1}$, Ran $Y=\operatorname{Ran} A=\operatorname{dom} Y^{-1}$, and $Y^{-1} Y$ and $Y Y^{-1}$ are the identity operators on $\operatorname{dom} Y$ and $\operatorname{dom} Y^{-1}$, respectively. 


$$
\begin{aligned}
L(x, y) & =\left[L_{y}, L_{x}\right]=\left(\left(Y^{*} Y\right)^{-1} \pi K_{y}, \pi K_{x}\right)_{\mathscr{E}} \\
& =\left(Y^{*-1} \pi K_{y}, Y^{*-1} \pi K_{x}\right)_{\mathscr{F}}
\end{aligned}
$$

(the first line makes sense only if $\pi K_{y} \in \operatorname{Ran} A^{*} A$, the second one holds in full generality).

As a first application of Theorem 4.1, let us give an alternative proof of the Lions formula (2.12) and its Neumann analogue (Theorem 3.1).

Another proof of Theorem 2.1. For simplicity of ideas, we present the proof only for the Laplace operator $L=\Delta$ with the Dirichlet boundary condition $B_{0}=I$. (This corresponds to the original Lions's formula in [L2].) Thus we are interested in the reproducing kernels $K(x, y)$ of the spaces

$$
\mathscr{H}^{s}(\Omega):=\left\{u \in \mathscr{D}^{\prime}(\bar{\Omega}): \Delta u=0, \gamma u \in H^{s}(\Gamma)\right\}
$$

with the scalar product

$$
(u, v)_{\mathscr{H}^{s}(\Omega)}:=(\gamma u, \gamma v)_{H^{s}(\Gamma)} .
$$

We want to show that

$$
K(x, y)=\left(M^{-s} P_{y}, P_{x}\right)_{L^{2}(\Gamma)} .
$$

Observe first of all that (4.5) is trivially satisfied for $s=0$. Indeed, by (2.8),

$$
u(x)=\left(\gamma u, P_{x}\right)_{L^{2}(\Gamma)}=\left(u, \mathbf{P} P_{x}\right)_{\mathscr{H}^{0}(\Omega)} \quad \forall u \in \mathscr{H}^{0}(\Omega),
$$

where we have put $P_{x}:=P_{x}^{(0)}$. Since the reproducing kernel $K_{x}$ is uniquely determined by the reproducing property $u(x)=\left(u, K_{x}\right)_{\mathscr{H}^{0}(\Omega)} \forall u$, we must have $K_{x}=\mathbf{P} P_{x}$. Thus

$$
K(x, y)=\left(K_{y}, K_{x}\right)_{\mathscr{H}^{0}(\Omega)}=\left(\mathbf{P} P_{y}, \mathbf{P} P_{x}\right)_{\mathscr{H}^{0}(\Omega)}=\left(P_{y}, P_{x}\right)_{L^{2}(\Gamma)},
$$

as claimed.

Now apply Theorem 4.1 with $\mathscr{E}=\mathscr{F}=\mathscr{H}^{0}(\Omega)$ and $A=\mathbf{P} M^{s / 2} \gamma$, with some $s \in \mathbb{R}$. Then $\operatorname{ker} A=\{0\}$, so

$$
\mathscr{M}_{A}=\operatorname{dom} A=\mathbf{P}\left(\operatorname{dom} M^{s / 2}\right)
$$

and

$$
[\mathbf{P} f, \mathbf{P} g]=\left(\mathbf{P} M^{s / 2} f, \mathbf{P} M^{s / 2} g\right)_{\mathscr{H}^{0}(\Omega)}=\left(M^{s} f, g\right)_{L^{2}(\Gamma)}=(f, g)_{H^{s}(\Gamma)}
$$

for all $\mathbf{P} f, \mathbf{P} g \in \mathscr{M}_{A}$. It follows that $\mathscr{E}_{A}$ must coincide with $\mathbf{P} H^{s}(\Gamma)=\mathscr{H}^{s}(\Omega)$ with the inner product (4.4).

Let us now see what Theorem 4.1 above says about the reproducing kernel for $\mathscr{E}_{A}$. Observe that $A$ is selfadjoint on $\mathscr{H}^{0}(\Omega)$ (since $M^{s / 2}$ is selfadjoint on $L^{2}(\Gamma)$ ) and $\pi$ is the 
identity in view of the injectivity of $A$. By the theorem, the point evaluations will thus be continuous on $\mathscr{E}_{A}$ if and only if $K_{x} \in \operatorname{Ran} A$, or

$$
P_{x} \in \operatorname{Ran} M^{s / 2}=\operatorname{dom} M^{-s / 2}
$$

for all $x \in \Omega$. As $P_{x} \in C^{\infty}(\Gamma)$, this clearly holds for any $s \in \mathbb{R}$, by $(2.1)$. Thus $\mathscr{E}_{A}$ has a reproducing kernel which, by (4.3), is given by

$$
\begin{aligned}
L(x, y) & =\left(A^{-1} K_{y}, A^{-1} K_{x}\right)_{\mathscr{H}^{0}(\Omega)}=\left(A^{-1} \mathbf{P} P_{y}, A^{-1} \mathbf{P} P_{x}\right)_{\mathscr{H}^{0}(\Omega)} \\
& =\left(\mathbf{P} M^{-s / 2} P_{y}, \mathbf{P} M^{-s / 2} P_{x}\right)_{\mathscr{H}^{0}(\Omega)}=\left(M^{-s / 2} P_{y}, M^{-s / 2} P_{x}\right)_{L^{2}(\Gamma)},
\end{aligned}
$$

which is the desired formula.

Another proof of Theorem 3.1. Take again $\mathscr{E}=\mathscr{F}=\mathscr{H}^{0}(\Omega)$, but now with $A=\mathbf{P} M^{(s-1) / 2} \gamma N$. This time $A$ has a nontrivial kernel: $\operatorname{ker} A=\mathbb{R} 1$, the subspace of constant functions. Thus $\pi$ is the orthogonal projection in $\mathscr{H}^{0}(\Omega)$ onto $1^{\perp}$. As in the previous proof, one sees that

$$
\begin{aligned}
{[\mathbf{P} f, \mathbf{P} g] } & =\left(\mathbf{P} M^{(s-1) / 2} \gamma N \mathbf{P} f, \mathbf{P} M^{(s-1) / 2} \gamma N \mathbf{P} g\right)_{\mathscr{H}^{0}(\Omega)} \\
& =\left(M^{s-1} \gamma N \mathbf{P} f, \gamma N \mathbf{P} g\right)_{L^{2}(\Gamma)} \\
& =(\gamma N \mathbf{P} f, \gamma N \mathbf{P} g)_{H^{s-1}(\Gamma)},
\end{aligned}
$$

for all $f, g \in \mathscr{M}_{A}$. It follows that $\mathscr{E}_{A}$ coincides with the space $\mathscr{H}_{\text {Neum }}^{s}(\Omega)$ from Theorem 3.1.

A brief computation reveals that $A^{*}=\mathbf{P} \gamma N \mathbf{P} M^{(s-1) / 2} \gamma$. Thus by Theorem 4.1, $\mathscr{E}_{A}$ has bounded point evaluations if and only if

$$
\pi \mathbf{P} P_{x} \in \operatorname{Ran} A^{*}=\mathbf{P} \gamma N \mathbf{P} H^{1-s}(\Gamma) \subset \mathbf{P} H^{-s}(\Gamma),
$$

which is, again, always the case as both $P_{x}$ and 1 belong to $C^{\infty}(\Gamma)$. Thus $\mathscr{E}_{A}$ admits a reproducing kernel given, by (4.3), by

$$
L(x, y)=\left(Y^{*-1} \pi \mathbf{P} P_{y}, Y^{*-1} \pi \mathbf{P} P_{x}\right)_{\mathscr{H}^{0}(\Omega)}=:\left(q_{y}, q_{x}\right)_{L^{2}(\Gamma)},
$$

where $q_{x}=\gamma Y^{*-1} \pi \mathbf{P} P_{x}$ is the element of $L^{2}(\Gamma)$ determined uniquely by

$$
\mathbf{P} q_{x} \perp 1 \text { and } A^{*} \mathbf{P} q_{x}=\pi \mathbf{P} P_{x}
$$

that is,

$$
q_{x} \perp 1 \quad\left(\text { in } L^{2}(\Gamma)\right) \quad \text { and } \quad \gamma N \mathbf{P} M^{(s-1) / 2} q_{x}=\pi_{2} P_{x}
$$

where $\pi_{2}$ denotes, for the moment, the orthogonal projection onto $1^{\perp}$ in $L^{2}(\Gamma)$.

We proceed to identify $\pi_{2} P_{x}$. Recall that the ordinary Green function $G(x, y) \equiv G_{y}(x)$ for the Laplace operator satisfies 


$$
\begin{array}{cc}
\Delta G_{y}=\delta_{y} & \text { on } \Omega, \\
\gamma G_{y}=0 & \text { on } \Gamma,
\end{array}
$$

and the corresponding Poisson kernel $P_{y}$ is given by (cf. Example 4.2 and (2.9))

$$
P_{y}=\gamma N G_{y} .
$$

Similarly, let $F(x, y) \equiv F_{y}(x)$ be the Neumann function for $\Delta$, as defined by (3.1) above, and let us also introduce the notation

$$
Q_{y}:=-\gamma F_{y}
$$

for the Neumann kernel (3.4) which solves the Neumann problem (3.2). Observe now that the difference $v_{x}:=G(\cdot, x)-F(\cdot, x)$ of the Green and the Neumann function-which is a harmonic function on $\Omega$, as the singularities of $G$ and $F$ cancel out-satisfies, in view of (4.7) and (3.1),

$$
\gamma v_{x}=-\gamma F(\cdot, x)=Q_{x}
$$

and

$$
\gamma N v_{x}=P_{x}+\frac{1}{\sigma(\Gamma)}
$$

The first of these equations implies that $v_{x}=\mathbf{P} Q_{x}$, and the second thus says that

$$
P_{x}=\gamma N \mathbf{P} Q_{x}-\frac{1}{\sigma(\Gamma)} .
$$

As $\operatorname{Ran} \gamma N \mathbf{P} \perp 1$, by Green's formula, we have thus shown that

$$
\pi_{2} P_{x}=\gamma N \mathbf{P} Q_{x}
$$

Substituting the last formula into (4.6), we get, using also the third condition in (3.1) and the fact that $M^{(s-1) / 2}$ preserves orthogonality to 1 (since $M$ is selfadjoint and $M 1=1$ ),

$$
q_{x}=M^{(1-s) / 2} Q_{x} .
$$

Consequently, the reproducing kernel is in this case given by

$$
L(x, y)=\left(q_{y}, q_{x}\right)_{L^{2}(\Gamma)}=\left(M^{1-s} Q_{y}, Q_{x}\right)_{L^{2}(\Gamma)}=\left(M^{1-s} \gamma F_{y}, \gamma F_{x}\right)_{L^{2}(\Gamma)},
$$

in complete agreement with Theorem 3.1.

In the rest of this section, we show how Theorem 4.1 can be used to recover some classical results of Bergman and Schiffer [BS], Garabedian [G2], and Zaremba [Z1].

Theorem 4.2. The reproducing kernel of the Dirichlet-type space 


$$
L_{\text {energy }}^{2}(\Omega):=\left\{u \in C^{\infty}(\Omega): \Delta u=0, \int_{\Omega}\|\nabla u\|^{2} d \tau<\infty, \int_{\Gamma} \gamma u d \sigma=0\right\}
$$

with the norm

$$
\|u\|^{2}:=\int_{\Omega}\|\nabla u\|^{2} d \tau=(\gamma N u, \gamma u)_{L^{2}(\Gamma)}
$$

is given by

$$
L(x, y)=G(x, y)-F(x, y),
$$

the difference between the Green and the Neumann functions.

Proof. Let us apply Theorem 4.1 with $\mathscr{E}=\mathscr{H}_{0}(\Omega)$ (again) and

$$
\mathscr{F}=L^{2}\left(\Omega, \mathbb{R}^{n}\right),
$$

the $L^{2}$-space on $\Omega$ of vector-valued functions with values in $\mathbb{R}^{n}$ (with respect to the Lebesgue measure), and

$$
A: \mathscr{H}^{0}(\Omega) \ni f \mapsto \nabla f \in L^{2}\left(\Omega, \mathbb{R}^{n}\right) .
$$

As in the alternative proof of Theorem 3.1, we have $\operatorname{ker} A=\mathbb{R} 1$, the subspace of constant functions in $\mathscr{H}^{0}(\Omega)$. For any $f$ in, say, $\mathbf{P} C^{\infty}(\Omega) \subset \operatorname{dom} A$, we have by the ordinary Green's formula

$$
(A f, A f)_{\mathscr{F}}=\int_{\Omega}\|\nabla f\|^{2} d \tau=(\gamma N f, \gamma f)_{L^{2}(\Gamma)} .
$$

The second equality, in conjunction with

$$
(A f, A f)_{\mathscr{F}}=\left(A^{*} A f, f\right)_{\mathscr{H}^{0}(\Omega)}=\left(\gamma A^{*} A f, \gamma f\right)_{L^{2}(\Gamma)},
$$

implies that

$$
A^{*} A=\mathbf{P} \gamma N
$$

while from the first it follows that $\mathscr{E}_{A}$ coincides with the space (4.10) with the norm given by (4.11).

Using the first equation in (4.3), we see that the reproducing kernel is given by

$$
L(x, y)=\left(\left(Y^{*} Y\right)^{-1} \pi \mathbf{P} P_{y}, \pi \mathbf{P} P_{x}\right)_{\mathscr{H}^{0}(\Omega)}=:\left(\mathbf{P} r_{y}, \pi \mathbf{P} P_{x}\right)_{\mathscr{H}^{0}(\Omega)},
$$

where $r_{y}=\gamma\left(Y^{*} Y\right)^{-1} \pi \mathbf{P} P_{y} \in L^{2}(\Gamma)$ satisfies

$$
r_{y} \perp 1, \quad A^{*} A \mathbf{P} r_{y}=\pi \mathbf{P} P_{y},
$$

that is, recalling (4.9) from the preceding proof and (4.12), 


$$
r_{y} \perp 1, \quad \gamma N \mathbf{P} r_{y}=\gamma N \mathbf{P} Q_{y} .
$$

Consequently, $r_{y}=Q_{y}$, and

$$
\begin{aligned}
L(x, y) & =\left(\mathbf{P} Q_{y}, \pi \mathbf{P} P_{x}\right)_{\mathscr{H}^{0}(\Omega)}=\left(\mathbf{P} Q_{y}, \mathbf{P} P_{x}\right)_{\mathscr{H}^{0}(\Omega)} \\
& =\left(Q_{y}, P_{x}\right)_{L^{2}(\Gamma)}=\mathbf{P} Q_{y}(x)=G(x, y)-F(x, y)
\end{aligned}
$$

(cf. the preceding alternative proof of Theorem 3.1). This completes the proof.

The last result agrees with the formula in [BS], Part B, Chapter II, §1, which however was derived there not for $\Delta$ but for $\Delta-q$, with $q>0$, so that the energy norm (4.11) was positive definite. The latter result is even easier to obtain by the same method as above than Theorem 4.2 (the corresponding operator $A$ is injective, hence the projection $\pi$ drops out) and we leave its details to the interested reader.

Theorem 4.3. The reproducing kernel of the harmonic Bergman space

$$
L_{\text {harm }}^{2}(\Omega):=\left\{u \in L^{2}(\Omega): \Delta u=0\right\},
$$

with the norm inherited from $L^{2}(\Omega)$, is equal to

$$
L(x, y)=\delta(x-y)-\Delta_{x} \Delta_{y} B(x, y),
$$

where $B(x, y) \equiv B_{y}(x)$ is the biharmonic Green function of $\Omega$, i.e. the solution of the boundary value problem

$$
\begin{aligned}
\Delta^{2} B_{y} & =\delta(\cdot-y) \quad(\text { the point mass at } y), \\
\gamma B_{y} & =\gamma N B_{y}=0 .
\end{aligned}
$$

Proof. Apply Theorem 4.1 with $\mathscr{E}=\mathscr{H}^{0}(\Omega)$ (again), $\mathscr{F}=L^{2}(\Omega)$, the ordinary Lebesgue space on $\Omega$, and $A$ the inclusion mapping from $\mathscr{H}^{0}(\Omega)$ into $\mathscr{F}$. For $u, v$ in, say, $\mathbf{P} C^{\infty}(\Gamma) \subset \operatorname{dom} A$, we then have

$$
[u, v]=(u, v)_{L^{2}(\Omega)},
$$

and it follows that $\mathscr{E}_{A}$ coincides with the (closed) subspace of harmonic functions in $L^{2}(\Omega)$, i.e. with $L_{\text {harm }}^{2}(\Omega)$. Also, since, on the one hand,

$$
\left(A^{*} A u, v\right)_{\mathscr{H}^{0}(\Omega)}=\left(\gamma A^{*} A u, \gamma v\right)_{L^{2}(\Gamma)},
$$

while, on the other hand,

$$
\left(A^{*} A u, v\right)_{\mathscr{H}^{0}(\Omega)}=(u, v)_{L^{2}(\Omega)}=(u, \mathbf{P} \gamma v)_{L^{2}(\Omega)}=\left(\mathbf{P}^{*} u, \gamma v\right)_{L^{2}(\Gamma)},
$$

where the adjoint $\mathbf{P}^{*}$ is taken with respect to the $L^{2}$ products on both $\Gamma$ and $\Omega$, we see that $\gamma A^{*} A=\mathbf{P}^{*}$, that is,

$$
A^{*} A=\mathbf{P P}^{*} .
$$


Finally, as $A$ is injective, the projection $\pi$ reduces to the identity. Applying the formula (4.3), we thus see that the reproducing kernel of $\mathscr{E}_{A}$ is given by

$$
L(x, y)=\left(\left(A^{*} A\right)^{-1} \mathbf{P} P_{y}, \mathbf{P} P_{x}\right)_{\mathscr{H}^{0}(\Omega)}=\left(\gamma\left(A^{*} A\right)^{-1} \mathbf{P} P_{y}, P_{x}\right)_{L^{2}(\Gamma)}=:\left(s_{y}, P_{x}\right)_{L^{2}(\Gamma)},
$$

where $s_{x} \in L^{2}(\Gamma)$ is determined by

$$
A^{*} A \mathbf{P} s_{x}=\mathbf{P} P_{x}
$$

or, using (4.14),

$$
\mathbf{P}^{*} \mathbf{P} s_{x}=P_{x}
$$

Let us now examine the adjoint $\mathbf{P}^{*}$. Since $\mathbf{P}$ is an integral operator with kernel $P_{x}(y)$, $\mathbf{P}^{*}$ is the integral operator with the conjugate kernel, i.e.

$$
\mathbf{P}^{*} f(x)=\int_{\Omega} f(y) P_{y}(x) d \tau(y)=\int_{\Omega} f(y) \gamma_{x} N_{x} G(x, y) d \tau(y)
$$

by (4.8), for any $x \in \Gamma$. It follows that

$$
\mathbf{P}^{*}=\gamma N \mathbf{G}
$$

where $\mathbf{G}$ is the integral operator on $L^{2}(\Omega)$ with kernel $G(x, y)$, i.e. $u=\mathbf{G} f$ is the solution to the Laplace equation $\Delta u=f$ with the Dirichlet boundary conditions $\gamma u=0$. By (4.15) we thus get

$$
\gamma N \mathbf{G P} s_{x}=P_{x}
$$

Consequently, the function $f_{x}:=\mathbf{G P} s_{x}$ satisfies

$$
\begin{aligned}
\Delta^{2} f_{x} & =\Delta \mathbf{P} s_{x}=0 & & \left(\text { i.e. } f_{x} \text { is biharmonic on } \Omega\right), \\
\gamma f_{x} & =0 & & \left(\text { since } f_{x} \in \operatorname{Ran} \mathbf{G}\right), \\
\gamma N f_{x} & =P_{x}, & &
\end{aligned}
$$

and

$$
\gamma \Delta f_{x}=\gamma \mathbf{P} s_{x}=s_{x}
$$

Recall now that, quite generally, the solution to the boundary value problem

$$
\begin{aligned}
\Delta^{2} u & =0, \\
\gamma u & =0, \\
\gamma N u & =f
\end{aligned}
$$

is given by 


$$
u(y)=\left(f,-\gamma \Delta B_{y}\right)_{L^{2}(\Gamma)},
$$

where $B(x, y) \equiv B_{y}(x)$ is the biharmonic Green function (cf. Example 2.4 above). Consequently, we obtain

$$
f_{x}(y)=\left(P_{x},-\gamma \Delta B_{y}\right)_{L^{2}(\Gamma)}
$$

and, hence,

$$
s_{x}(y)=\gamma_{y} \Delta_{y} f_{x}(y)=\left(P_{x},-\gamma \Delta \gamma_{y} \Delta_{y} B_{y}\right)_{L^{2}(\Gamma)} .
$$

To express this more neatly, observe that, by (4.13),

$$
\Delta_{x} \Delta_{y} B(x, y)=\delta(x-y)+h(x, y),
$$

where $h(x, y) \equiv h_{y}(x)$ is a function on $\Omega \times \Omega$ which is harmonic there in both $x$ and $y$. Thus

$$
\left(P_{x},-\gamma \gamma_{y} \Delta \Delta_{y} B_{y}\right)_{L^{2}(\Gamma)}=\left(P_{x},-\gamma \gamma_{y} h_{y}\right)_{L^{2}(\Gamma)}=-\mathbf{P} \gamma\left(\gamma_{y} h_{y}\right)(x)=-\gamma_{y} h(x, y)=-\gamma_{y} h(y, x),
$$

as $h$ is symmetric in $x$ and $y$. Consequently,

$$
s_{x}=-\gamma h_{x}, \quad \text { or } \quad \mathbf{P} s_{x}=-h_{x} .
$$

Substituting this, finally, into the formula for $L(x, y)$, we arrive at

$$
\begin{aligned}
L(x, y) & =\left(s_{y}, P_{x}\right)_{L^{2}(\Gamma)} \\
& =-\left(\gamma h_{y}, P_{x}\right)_{L^{2}(\Gamma)} \\
& =-\mathbf{P} \gamma h_{y}(x) \\
& =-h(x, y),
\end{aligned}
$$

which is the desired result.

The formula in Theorem 4.3 is due to Garabedian ([G2], pp. 265-267). Using the general Green formula (2.3), it is possible to obtain the analogous result for the space

$$
L_{L}^{2}(\Omega):=\left\{u \in L^{2}(\Omega): L u=0\right\},
$$

with the norm inherited from $L^{2}(\Omega)$, for any elliptic operator $L$ such that the Dirichlet problem for the operator $L L^{+}$is determinate. Namely, the reproducing kernel of $L_{L}^{2}(\Omega)$ is equal to

$$
\delta(x-y)-L_{x}^{+} L_{y}^{+} J(x, y),
$$


where $J(x, y) \equiv J_{y}(x)$ is the solution to the boundary value problem

$$
L L^{+} J_{y}=\delta_{y}, \quad \gamma N^{j} J_{y}=0 \quad \forall j=0,1, \ldots, 2 m-1 .
$$

The details are again left to the reader.

As a final application of Theorem 4.1 we recover a result of Zaremba ([Z1], p. 150; cf. also [L1]).

Theorem 4.4. The reproducing kernel of the space

$$
L_{\text {Dir }}^{2}(\Omega):=\left\{u \in C^{\infty}(\Omega): \Delta u=0,\|u\|_{D}^{2}:=\int_{\Omega}\|\nabla u\|^{2} d \tau<\infty, \int_{\Omega} u d \tau=0\right\},
$$

with the norm $\|u\|_{D}$ (one might call this the harmonic Dirichlet space on $\Omega$ ), equals $t(x, y)$, where $t(x, y) \equiv t_{y}(x)$ is the solution to the boundary value problem

$$
\begin{array}{rlrl}
\Delta t_{y} & =0 & & \text { on } \Omega, \\
\gamma N t_{y} & =P_{y}-\frac{1}{\tau(\Omega)} \gamma N \mathbf{G 1} & & \text { on } \Gamma, \\
\int_{\Omega} t_{y} d \tau & =0 . &
\end{array}
$$

Proof. This time we apply Theorem 4.1 with $\mathscr{E}$ not $\mathscr{H}^{0}(\Omega)$ but the harmonic Bergman space $L_{\text {harm }}^{2}(\Omega)$ from the preceding theorem, with $\mathscr{F}$ the vector-valued $L^{2}$ space $L^{2}\left(\Omega, \mathbb{R}^{n}\right)$ from Theorem 4.2, and $A$ the same operator $f \mapsto \nabla f$ as in Theorem 4.2. In the same way as before, one then sees that the corresponding space $\mathscr{E}_{A}$ can be identified with $L_{\text {Dir }}^{2}(\Omega)$ with the norm $\|u\|_{D}$. By (4.3), the reproducing kernel is given by

$$
L(x, y)=\left(\left(Y^{*} Y\right)^{-1} \pi \mathbf{P}_{y}, \pi \mathbf{P} s_{x}\right)_{L^{2}(\Omega)}=:\left(t_{y}, \mathbf{P s}_{x}\right)_{L^{2}(\Omega)},
$$

where $I-\pi$ is the orthogonal projection onto the constants in $L_{\text {harm }}^{2}(\Omega), s_{x}$ has the same meaning as in the previous proof (i.e. $\mathbf{P}_{x}(y)=-h(x, y)$ is the reproducing kernel of $\left.L_{\text {harm }}^{2}(\Omega)\right)$, and $t_{x} \in L_{\text {harm }}^{2}(\Omega)$ are uniquely determined by the conditions

$$
t_{x} \perp 1 \quad\left(\text { in } L^{2}(\Omega)\right), \quad A^{*} A t_{x}=\pi \mathbf{P} s_{x} .
$$

From the computation

$$
\left(\mathbf{P}^{*} A^{*} A u, \gamma v\right)_{L^{2}(\Gamma)}=\left(A^{*} A u, v\right)_{L^{2}(\Omega)}=(\nabla u, \nabla v)_{L^{2}\left(\Omega, \mathbb{R}^{n}\right)}=(\gamma N u, \gamma v)_{L^{2}(\Gamma)}
$$

we see that $\mathbf{P}^{*} A^{*} A u=\gamma N u$, or, using the notation $\mathbf{G}$ from the previous proof,

$$
\gamma N u=\gamma N \mathbf{G} A^{*} A u
$$

for all $u$ in, say, $\mathbf{P} C^{\infty}(\Gamma)$. Thus (4.18) implies that 


$$
\begin{aligned}
\gamma N t_{x} & =\gamma N \mathbf{G} \pi \mathbf{P} s_{x} \\
& =\gamma N \mathbf{G}\left(\mathbf{P} s_{x}-c_{x} 1\right) \\
& =\gamma N \mathbf{G P} s_{x}-c_{x} \gamma N \mathbf{G} 1 \\
& =P_{x}-c_{x} \gamma N \mathbf{G} 1 \quad(\text { by }(4.16)),
\end{aligned}
$$

and $t_{x} \perp 1$. Here

$$
c_{x}=\frac{\left(\mathbf{P}_{x}, 1\right)_{L^{2}(\Omega)}}{(1,1)_{L^{2}(\Omega)}}=\frac{1(x)}{\tau(\Omega)}=\frac{1}{\tau(\Omega)} .
$$

Finally, since $\mathbf{P} s_{x}(y)$ is the reproducing kernel for $L_{\text {harm }}^{2}(\Omega)$, we have

$$
\left(t_{y}, \mathbf{P}_{s_{x}}\right)_{L^{2}(\Omega)}=t_{y}(x)
$$

and the assertion follows.

Remark 4.5. We leave it to the reader to check that in all the applications above the operators $A$ indeed were densely defined and closed, as we had assumed them to be at the beginning.

\section{Some concrete examples}

In this section we consider some examples of reproducing kernels. All the domains $\Omega$ have sufficiently many symmetries so that the method of separation of variables, or the method of Fourier, is easily applicable. In addition we consider only the case of two dimensions (except in the last Section 5.6). But all our examples can in principle be extended to any number of dimensions.

Recall from the Introduction that the reproducing kernel in the Hilbert space $\mathscr{H}^{s}(\Omega)$, as defined there, is given by the formula (1.8)

$$
K(x, b)=\sum_{v} \hat{e}_{v}(x) \hat{e}_{v}(b)=\sum_{v} \lambda_{v}^{-s} e_{v}(x) e_{v}(b)
$$

where $e_{v}$ is an orthonormal basis of eigenfunctions of the "smoothed" Laplace-Beltrami operator $M$ on $\Gamma$ in the Hilbert space $L^{2}(\Gamma)$.

Remark 5.1. If the eigenfunctions in (5.1) are only orthogonal, but not necessarily orthonormal, then we have first to divide each term with the norm squares $\left\|e_{v}\right\|_{L^{s}(\Gamma)}^{2}$. As we already remarked in the Introduction (Remark 1.2), in the case of complex scalars, the second factor in each term of the series in (5.1) has to be taken with its complex conjugate.

5.1. The disk. Let $\Omega=\mathbb{D}=$ the (open) unit disk with boundary $\Gamma=\mathbb{T}=$ unit circle. We are going to use polar coordinates writing $x=(r \cos \theta, r \sin \theta)$ with $r=|x|=\sqrt{x_{1}^{2}+x_{2}^{2}}, \theta=\arg x$. In this case we have 


$$
\Lambda=-D^{2}=-\frac{d^{2}}{d \theta^{2}} ; \quad D=\frac{d}{d \theta}
$$

so that

$$
M=-D^{2}+1=-\frac{d^{2}}{d \theta^{2}}+1
$$

As a basis we can take Fourier's own system, that is, the functions whose restriction to $\mathbb{T}$ is given by

$$
\frac{1}{\sqrt{2}} ; \quad \cos \theta ; \quad \sin \theta ; \quad \cos 2 \theta ; \quad \sin 2 \theta ; \quad \ldots
$$

The corresponding eigenvalues are

$$
\begin{array}{ccccccc}
D: & 0 ; & 1 ; & 1 ; & 2 ; & 2 ; & \ldots \\
M: & 1 ; & 2 ; & 2 ; & 5 ; & 5 ; & \ldots
\end{array}
$$

(To an eigenvalue $n$ of $D$ there corresponds the eigenvalue $n^{2}+1$ of $M$.)

Harmonic continuation gives the functions

$$
\frac{1}{\sqrt{2}} ; \quad r \cos \theta ; \quad r \sin \theta ; \quad r^{2} \cos 2 \theta ; \quad r^{2} \sin 2 \theta ; \quad \ldots
$$

They are however not orthonormalized, the square of the norm of each of them being equal to $\pi$.

Using (5.1), along with the recipe in Remark 5.1, we find

$$
K(x, b)=\frac{1}{\pi}\left(\frac{1}{2}+\sum_{n=1}^{\infty} r^{n} \rho^{n}\left(n^{2}+1\right)^{-s}(\cos n \theta \cos n \beta+\sin n \theta \sin n \beta)\right)
$$

where again we have put $\rho=|b|$ and $\beta=\arg b$. The sum in (5.2) can also be written more simply as

$$
\sum_{n=1}^{\infty} r^{n} \rho^{n}\left(n^{2}+1\right)^{-s} \cos n(\theta-\beta)
$$

i.e., as a function of the difference $\theta-\beta$, which again is a reflection of the fact that we have a rotation invariant situation.

Remark 5.2. If we "cheat" a little bit and replace the factor $n^{2}+1$ by only $n^{2}$, the series will be somewhat easier to handle. If $S=2 s$ is an integer $>0$ (or $s$ a half-integer), one encounters then for $r=\rho=1$ Bernoulli functions in (5.2). 
Note that the Bernoulli functions satisfy the differential equation $D^{S} u=\delta_{\text {per }}$, where $\delta_{\text {per }}$ is the "periodic" delta function, the sum of ordinary point masses placed at the integer multiples $2 \pi m$ of $2 \pi$. It follows that the restriction of $u$ to an interval between two consecutive such points is a polynomial of degree $S$. Taking the interval $(0,2 \pi)$ gives the Bernoulli polynomials. However, in the standard normalization one takes the period to be 1 , not $2 \pi$.

In the general case one has thus to deal with a kind generalized such functions. If one considers more generally instead of $M$ the operators $M_{\varepsilon}=-D^{2}+\varepsilon$ and makes $\varepsilon \rightarrow 0$, the usual Bernoulli functions appear in the "partie finie".

It remains to compare this with Lions's formula (1.12). To this end consider the Poisson kernels

$$
P(y, x)=P_{x}(y)=N_{y} G(y, x)
$$

and analogously

$$
P(y, b)=P_{b}(y)=N_{y} G(y, b)
$$

(These are instances of the most common Poisson kernel.) Writing $\phi=\arg y$ one obtains the Fourier expansions

$$
P(y, x)=\frac{1}{\pi}\left(\frac{1}{2}+\sum_{n=1}^{\infty} r^{n}(\cos n \theta \cos n \phi+\sin n \theta \sin n \phi)\right),
$$

respectively

$$
P(y, b)=\frac{1}{\pi}\left(\frac{1}{2}+\sum_{n=1}^{\infty} q^{n}(\cos n \beta \cos n \phi+\sin n \beta \sin n \phi)\right) .
$$

Next, we apply the operators $M_{y}^{-\frac{s}{2}}$. Then the factor $\left(n^{2}+1\right)^{-s / 2}$ appears. If we multiply these expressions with each other and $d \phi$, moreover integrating over $(0,2 \pi)$ and taking care of the orthogonality, we obtain the left hand side of [L2], (2.13). In other words, we have proved anew Lions's formula (1.12) in this special case.

5.2. The half plane. We consider the plane $\mathbb{R}^{2}$ with points now denoted by bold face letters $\boldsymbol{x}=\left(x_{1}, x_{2}\right)$, where we at once set $x_{1}=x, x_{2}=t$ - this in order to avoid lots of disturbing subscripts. Let $\Omega=\{t>0\}$ be the north (or upper) half plane. We further consider tempered harmonic functions $u=u(x, t)$ in $\Omega$ with a tempered trace on the boundary $\partial \Omega=\mathbb{R}$. By $\hat{u}(\xi, t)$ we designate the partial Fourier transform of $u$ with respect to $x$. (We denote the variable dual to $x$ by $\xi$.) From

$$
\frac{\partial^{2} u}{\partial x^{2}}+\frac{\partial^{2} u}{\partial t^{2}}=0
$$

it follows that

$$
-\xi^{2} \hat{u}+\frac{\partial^{2} \hat{u}}{\partial t^{2}}=0
$$


Hence

$$
\hat{u}(\xi, t)=e^{-t|\xi|} \hat{u}(\xi)
$$

where we, for the sake of simplicity, have put $\hat{u}(\xi)=\hat{u}(\xi, 0)$. In other words, one can identify $u(x, t)$ with $\hat{u}(\xi)$. Recall that, quite generally, the Fourier transform of a function or a distribution $v(x)$ on $\mathbb{R}$ is formally given by

$$
\hat{v}(\xi)=\int_{\mathbb{R}} e^{-i x \xi} v(x) d x .
$$

Next we introduce the complex Hilbert space $H^{s}(\mathbb{R})$ with norm

$$
\|u\|_{s}^{2}=\frac{1}{2 \pi} \int_{-\infty}^{\infty}\left(1+\xi^{2}\right)^{s}|\hat{u}(\xi)|^{2} d \xi
$$

and inner product

$$
(u, v)_{s}=\frac{1}{2 \pi} \int_{-\infty}^{\infty}\left(1+\xi^{2}\right)^{s} \hat{u}(\xi) \overline{\hat{v}(\xi)} d \xi
$$

Similarly, we let $\mathscr{H}^{s}(\Omega)$ denote the space of harmonic distributions $u$ whose traces on $\mathbb{R}$ belong to $H^{s}(\mathbb{R})$. In fact, it holds, in these identifications, $\mathscr{H}^{s}(\Omega)=$ the Sobolev space $H^{s}(\mathbb{R})$. formula,

Consider further an auxiliary point $\boldsymbol{b}=(b, a) \in \Omega$. In view of Fourier's inversion

$$
u(\boldsymbol{b})=u(b, a)=\frac{1}{2 \pi} \int_{-\infty}^{\infty} e^{i b \xi} e^{-a|\xi|} \hat{u}(\xi) d \xi
$$

We rewrite this formula as

$$
u(\boldsymbol{b})=u(b, a)=\frac{1}{2 \pi} \int_{-\infty}^{\infty}\left(1+\xi^{2}\right)^{s} \hat{u}(\xi) \frac{\overline{e^{-i b \xi} e^{-a|\xi|}}}{\left(1+\xi^{2}\right)^{s}} d \xi
$$

This has the form of a complex (!) inner product. From here we can read off the reproducing kernel: We have

$$
u(\boldsymbol{b})=(u, K(\boldsymbol{x}, \boldsymbol{b}))_{s}
$$

with

$$
K(\boldsymbol{x}, \boldsymbol{b})=K(x, t ; b, a)=\frac{1}{2 \pi} \int_{-\infty}^{\infty} e^{i x \xi} \frac{e^{-i b \xi} e^{-a|\xi|-t|\xi|}}{\left(1+\xi^{2}\right)^{s}} d \xi \text {. }
$$


Observe that from (5.5), which is our main formula, it is seen that $K(\boldsymbol{x}, \boldsymbol{b})$ depends only on the quantities $x-b$ and $t+a$. Then dependence on the difference $x-b$ was expected, it is just a reflection of the fact that we are in a translation invariant situation. But the dependence on the sum $t+a$ is a little bit more mysterious.

This observation leads us to introduce the function

$$
\mathfrak{F}(x, t)=\mathfrak{F}_{s}(x, t)=\frac{1}{2 \pi} \int_{-\infty}^{\infty} e^{i x \xi} \frac{e^{-t|\xi|}}{\left(1+\xi^{2}\right)^{s}} d \xi
$$

With this definition (5.6) can (5.5) be written more compactly as

$$
K(x, t ; b, a)=\mathfrak{F}(x-b, t+a) .
$$

Remark 5.3. The integral in (5.7) is, up to a factor, the modified Bessel function of the second kind, or Macdonald function $K_{v}$, with index $v=s-(1 / 2)$. (For this function see [MOS], Section 3.1 (p. 65) and [W], Section 3.7 (p. 78).) This relation can be found in [MOS], p. 400 and [W], p. 172. In the latter place it is named after Basset 1888/1889. The proof given there is obtained as a result of a complicated chain of "contour integrations". Some other famous names mentioned there ([W], p. 173) include: Poisson 1813; Catalan 1840; Serret 1843. Another, direct proof can be given by first verifying that $\mathfrak{F}$ satisfies the modified Bessel equation and then identifying the constants.

Note that the generalized Bernoulli functions indicated in Remark 5.2 could be viewed as periodic counterparts of the Macdonald function $K_{v}$. Do they possess properties reminiscent to the ones of that function?

Next, let $\boldsymbol{y}=(y, 0)$ be an auxiliary point on the boundary of $\Omega(=\mathbb{R})$. Then we can write down the two Poisson kernels

$$
P(\boldsymbol{y}, \boldsymbol{x})=\frac{1}{2 \pi} \int_{-\infty}^{\infty} e^{i(x-y) \xi} e^{-t|\xi|} d \xi
$$

and

$$
P(\boldsymbol{y}, \boldsymbol{b})=\frac{1}{2 \pi} \int_{-\infty}^{\infty} e^{i(b-y) \eta} e^{-a|\eta|} d \eta
$$

We compose them with the operator $\left(1-\Delta_{y}\right)^{-\frac{s}{2}}$. This yields

$$
\left(1-\Delta_{y}\right)^{-\frac{s}{2}} P(\boldsymbol{y}, \boldsymbol{x})=\frac{1}{2 \pi} \int_{-\infty}^{\infty}\left(1+\xi^{2}\right)^{-\frac{s}{2}} e^{i(x-y) \xi} e^{-t|\xi|} d \xi
$$

and

$$
\left(1-\Delta_{y}\right)^{-\frac{s}{2}} P(\boldsymbol{y}, \boldsymbol{b})=\frac{1}{2 \pi} \int_{-\infty}^{\infty}\left(1+\eta^{2}\right)^{-\frac{s}{2}} e^{i(b-y) \eta} e^{-a|\eta|} d \eta
$$


We now want to integrate the product of these two functions with respect to $y$. In doing this we take account of the following orthogonality relation:

$$
\frac{1}{2 \pi} \int_{-\infty}^{\infty} e^{i x \xi} \int_{-\infty}^{\infty} e^{i y \eta} d y=\delta(\xi-\eta) \quad \text { (the Fourier-Gel'fand formula); }
$$

concerning Fourier's contribution to it see [F], nr. 361, p. 351, and [Pe4].

This gives

$$
\int_{-\infty}^{\infty}\left(1-\Delta_{y}\right)^{-\frac{s}{2}} P(\boldsymbol{y}, \boldsymbol{x}) \cdot\left(1-\Delta_{y}\right)^{-\frac{s}{2}} P(\boldsymbol{y}, \boldsymbol{b}) d y=K(\boldsymbol{x}, \boldsymbol{b}),
$$

which apparently is the counterpart to Lions's formula in our special case.

Remark 5.4. Observe that (5.8) is just a concise way of expressing Fourier's inversion formula. If one does not like distributions and delta functions one can for sure replace the above somewhat heuristic computation by a more formal proof based on this formula.

5.3. The case of an annulus. In this section we turn to a case when the boundary is not connected but consists of two pieces. More precisely, we take $\Omega$ to be an annulus in the plane bounded by two concentric circles $\Gamma_{0}$ and $\Gamma_{1}$. We may assume that the radii are $R<1$ and 1 respectively, and moreover place the center at the origin. As before we consider the Hilbert space $\mathscr{H}^{s}(\Omega)$ of distributions harmonic in $\Omega$ with trace on $\Gamma$ belonging to the Sobolev space $H^{s}(\Gamma)$. Now $H^{s}(\Gamma)$ comes as the orthogonal sum of two closed subspaces $H_{0}^{s}(\Gamma)$ and $H_{1}^{s}(\Gamma)$, where $H_{0}^{s}(\Gamma)$ consists of those elements of $H^{s}(\Gamma)$ which vanish on $\Gamma_{1}$ and, vice versa, $H_{1}^{s}(\Gamma)$ consists of those elements of $H^{s}(\Gamma)$ which vanish on $\Gamma_{0}$ :

$$
H^{s}(\Gamma)=H_{0}^{s}(\Gamma) \oplus H_{1}^{s}(\Gamma)
$$

Corresponding to this decomposition $(5.10)$ the space $\mathscr{H}^{s}(\Omega)$ has the decomposition

$$
\mathscr{H}^{s}(\Omega)=\mathscr{H}_{0}^{s}(\Omega) \oplus \mathscr{H}_{1}^{s}(\Omega) .
$$

Thus e.g. $\mathscr{H}_{0}^{s}(\Omega)$ consists of the elements of $\mathscr{H}^{s}(\Omega)$ which vanish on $\Gamma_{1}$; similarly for $\mathscr{H}_{1}^{S}(\Omega)$.

Now, quite generally, let $H$ be a Hilbert space of functions on some space $\Omega$ which comes as the orthogonal sum of two closed subspaces $H_{1}$ and $H_{2}: H=H_{1} \oplus H_{2}$; let $K_{1}$ be the reproducing kernel of $H_{1}$ and $K_{2}$ be the one of $H_{2}$. Then, by some general nonsense, the reproducing kernel $K$ of $H$ is given by the orthogonal sum of $K_{1}$ and $K_{2}$ :

$$
K=K_{1}+K_{2}
$$

Let us return to our case. In view of (5.11) and (5.12) it suffices to determine the re- 
producing kernel of $\mathscr{H}_{0}^{s}(\Omega)$ and $\mathscr{H}_{1}^{s}(\Omega)$ separately. Let these two kernels again be written $K_{1}$ and $K_{2}$.

Let us uncover the structure of, say, the space $\mathscr{H}_{0}^{s}(\Omega)$, in particular, looking for an orthogonal basis. To this end consider first the functions

$$
u_{n}=r^{n} \cos n \theta-C r^{-n} \cos n \theta \quad(n=1,2, \ldots),
$$

where $C$ is a constant at our disposal. We wish to determine $C$ so that $u_{n}$ vanishes on $\Gamma_{1}$. So putting $r=R$ in (5.13) and putting the ensuing expression equal to zero gives $R^{n}-C R^{-n}=0$ or $C=R^{2 n}$. With this choice of $C$ formula (5.13) gets replaced by

$$
u_{n}=\left(r^{n}-R^{2 n} r^{-n}\right) \cos n \theta \quad(n=1,2, \ldots)
$$

In the same way, replacing the cosine by sine, we obtain the functions

$$
v_{n}=\left(r^{n}-R^{2 n} r^{-n}\right) \sin n \theta \quad(n=1,2, \ldots) .
$$

Apparently, the functions $u_{n}$ and $v_{n}$ are orthogonal. However, the resulting system is not yet complete. (Notice that the identities (5.14) and (5.15), for $n=0$, formally give the result zero!) To obtain a complete orthogonal system we have to add to them the function

$$
u_{0}=1-\frac{\log r}{\log R} .
$$

Note that this is a harmonic function with a logarithmic singularity at the origin and at infinity. Moreover, it is rotation invariant (radial). Then we have all right an orthogonal basis consisting of the one function $u_{0}$ and the infinitely many functions $u_{n}$ and $v_{n}$ $(n=1,2, \ldots)$. Here are their norm squares:

$$
\left\|u_{0}\right\|_{L^{2}\left(\Gamma_{0}\right)}^{2}=2 \pi ; \quad\left\|u_{n}\right\|_{L^{2}\left(\Gamma_{0}\right)}^{2}=\left\|v_{n}\right\|_{L^{2}\left(\Gamma_{0}\right)}^{2}=\pi\left(1-R^{2 n}\right)^{2} \quad(n=1,2, \ldots) .
$$

Using formula (5.1), and remembering to first divide with their norm squares (as indicated in Remark 5.1), we obtain for the reproducing kernel of $\mathscr{H}_{0}^{s}(\Omega)$ the expression

$$
\begin{aligned}
K_{0}(x, b)= & \frac{1}{2 \pi}\left(1-\frac{\log r}{\log R}\right)\left(1-\frac{\log \rho}{\log R}\right) \\
& +\frac{1}{\pi} \sum_{n=1}^{\infty} \frac{\left(n^{2}+1\right)^{-s}}{\left(1-R^{2 n}\right)^{2}}\left(r^{n}-R^{2 n} r^{-n}\right)\left(\rho^{n}-R^{2 n} \rho^{-n}\right) \cos n(\theta-\beta),
\end{aligned}
$$

where $(r, \theta)$ and $(\rho, \beta)$ are the polar coordinates of the point $x$ and $b$ respectively (cf. Section 5.1) and where we have used the subtraction theorem for cosine.

In the case of the space $\mathscr{H}_{1}^{s}(\Omega)$ we use instead the functions

$$
u_{0}^{*}=\ln r ; \quad u_{n}^{*}=\left(r^{-n}-r^{n}\right) \cos n \theta ; \quad v_{n}^{*}=\left(r^{-n}-r^{n}\right) \sin n \theta
$$

$(n=1,2, \ldots)$. The corresponding norm squares are 


$$
\left\|u_{0}^{*}\right\|_{L^{2}\left(\Gamma_{1}\right)}^{2}=2 \pi(\ln R)^{2} ; \quad\left\|u_{n}^{*}\right\|_{L^{2}\left(\Gamma_{1}\right)}^{2}=\left\|v_{n}^{*}\right\|_{L^{2}\left(\Gamma_{1}\right)}^{2}=\pi\left(R^{-n}-R^{n}\right)^{2} .
$$

This yields

$$
\begin{aligned}
K_{1}(x, b)= & \frac{1}{2 \pi} \frac{(\ln r)^{2}}{(\ln R)^{2}} \\
& +\frac{1}{\pi} \sum_{n=1}^{\infty} \frac{\left(n^{2}+1\right)^{-s}}{\left(R^{-n}-R^{n}\right)^{2}}\left(r^{-n}-r^{n}\right)\left(\rho^{-n}-\rho^{n}\right) \cos n(\theta-\beta) .
\end{aligned}
$$

Adding up (5.18) and (5.19) we get the desired formula for $K(x, b)$, which we, however, do not bother to write down, but we urge the Reader to do it.

It is an easy, though rather dull exercise to verify that in the limit $(R=0)$ one gets back the result of Section 5.1. (Use that $\left(\lim (\ln R)^{2}\right)^{-1}=0$.)

5.4. The heat equation. Exactly the same technique can be applied to other equations, e.g. take instead of (5.4) the heat equation

$$
\frac{\partial^{2} u}{\partial x^{2}}-\frac{\partial u}{\partial t}=0
$$

Then one has caloric functions (or temperatures) instead of harmonic ones. One obtains then from (5.1) a formula entirely analogous to (5.5). One has just to replace everywhere the factor $e^{-t|\xi|}$ by $e^{-t \xi^{2}}$. Let $\mathscr{K}^{s}(\Omega)$ denote the space of temperatures $u$ whose traces on $\mathbb{R}$ belong to the Sobolev space $H^{s}(\Gamma)$. Then its reproducing kernel, again denoted by the generic letter $K$, is given by

$$
K(\boldsymbol{x}, \boldsymbol{b})=K(x, t ; b, a)=\frac{1}{2 \pi} \int_{-\infty}^{\infty} e^{i x \xi} \frac{e^{-i b \xi} e^{-a \xi^{2}-t \xi^{2}}}{\left(1+\xi^{2}\right)^{s}} d \xi .
$$

Introducing further the function

$$
\mathfrak{W}(x, t)=\mathfrak{W}_{s}(x, t)=\frac{1}{2 \pi} \int_{-\infty}^{\infty} e^{i x \xi} \frac{e^{-t \xi^{2}}}{\left(1+\xi^{2}\right)^{s}} d \xi
$$

we can again write (5.22) more compactly as

$$
K(x, t ; b, a)=\mathfrak{6}(x-b, t+a) .
$$

Consider the case $s=0$ and thus the function $\mathfrak{6}_{0}$. Then the integral in (5.23) can be evaluated explicitly and one finds

$$
\mathfrak{5}_{0}(x, t)=\frac{1}{\sqrt{4 \pi t}} \exp \left(-\frac{x^{2}}{4 t}\right) .
$$


This is the famous heat kernel or Gauss-Weierstrass function ${ }^{9)}$. As is well-known, convolution with $\mathbf{6}$ gives the solution to the initial value problem for the equation (5.21).

Remark 5.5. Note that in our case $\mathfrak{5}_{0}(x-b, t)$ is going to play the rôle of the previous Poisson kernel.

Remark 5.6. For $s$ a positive integer, $\mathfrak{G}_{s}$ appears to be expressible in terms of the error function erf. For instance, for $s=1$ one has

$$
\mathfrak{G}_{1}(x, 1)=\frac{\sqrt{\pi}}{2}\left[e^{1-x} \operatorname{erf}\left(\frac{x}{2}-1\right)-e^{1+x} \operatorname{erf}\left(\frac{x}{2}+1\right)\right] .
$$

Remark 5.7. Imitating the procedure indicated at the end of Remark 5.3 we derived an ordinary linear differential equation for the function $\mathfrak{6}_{s}$ in (5.23). It is of the type called Laplace equation in Kamke's wonderful book [K], no. 22.4 of Part I, i.e. its coefficients are of the first order in the independent variable. As indicated there such equations can, in principle, be solved by quadratures using Laplace's method. However, our particular equation does not appear in Kamke's list [K], Chap. 3 of Part III, devoted to linear third order equations. So we have doubts that our function $\mathbf{6}$, for general $s$, admits a simple expression in terms of known special functions. Anyhow, the function $\mathfrak{b}_{s}$, for $s$ general, may be viewed as a generalization of the Gauss-Weierstrass function.

5.5. The heat equation (periodic solutions). We still consider the heat equation, viz. (5.21), but we consider only periodic solutions $u$. For convenience we take the period to be $2 \pi$. If such a solution $u$ has a trace on the boundary of $\Omega$, we have the Fourier representation

$$
u(x, t)=\frac{1}{2 \pi} \sum_{n=-\infty}^{\infty} A_{n} e^{i n x} e^{-n^{2} t}
$$

Formally taking $t=0$ gives the corresponding expansion of the trace:

$$
u(x, 0)=\frac{1}{2 \pi} \sum_{n=-\infty}^{\infty} A_{n} e^{i n x}
$$

We introduce the Hilbert space $\mathscr{K}_{\text {per }}^{s}(\Omega)$ of $2 \pi$-periodic temperatures $u$ corresponding to the metric

$$
\|u\|_{s}^{2}=\frac{1}{2 \pi} \sum_{n=-\infty}^{\infty}\left(1+n^{2}\right)^{s}\left|A_{n}\right|^{2}
$$

For any $u \in \mathscr{K}_{\text {per }}^{s}(\Omega)$ consider its evaluation at the point $(y, q)$ :

9) The name of Weierstrass is appended, probably, because the latter used this kernel in his original proof of the famous Weierstrass approximation theorem published in 1885. Indeed, Weierstrass possessed all the essential ideas about the Dirac delta function, in the guise of "approximate identities". 


$$
u(y, q)=\frac{1}{2 \pi} \sum_{n=-\infty}^{\infty} A_{n} e^{i n y} e^{-n^{2} q}
$$

Once more we try to rewrite this as an inner product:

$$
u(y, q)=\frac{1}{2 \pi} \sum_{n=-\infty}^{\infty}\left(1+n^{2}\right)^{s} a_{n} \times \frac{\overline{e^{i n y} e^{-n^{2} q}}}{\left(1+n^{2}\right)^{s}}
$$

Comparing this with formula (5.25) we obtain the reproducing kernel in $\mathscr{H}_{\text {per }}^{s}(\Omega)$ :

$$
K(x, t ; b, a)=K_{s}(x, t ; b, a)=\frac{1}{2 \pi} \sum_{n=-\infty}^{\infty} e^{i n x} e^{-n^{2} t} \frac{e^{-i n b} e^{-n^{2} a}}{\left(1+n^{2}\right)^{s}} .
$$

Again introducing the notation

$$
\Theta(x, t)=\Theta_{s}(x, t)=\frac{1}{2 \pi} \sum_{n=-\infty}^{\infty}\left(1+n^{2}\right)^{-s} e^{i n x} e^{-n^{2} t},
$$

we can rewrite $(5.26)$ as

$$
K(x, t ; b, a)=\Theta(x-b, t+a) .
$$

Remark 5.8. Apparently, $\Theta_{s}$ is the $2 \pi$-periodic analogue of the function $\boldsymbol{6}_{s}$ in Section 5.3. The case $s=0$ is of special interest, i.e. the function $\Theta_{0}$. Indeed, up to normalization it is one of Jacobi's four "elliptic" theta functions, to wit the one denoted $\theta_{3}$ in $[\mathrm{MOS}]$, Section 10.2, one of the most important special functions in mathematics. (They were encountered by Jacobi in his Fundamentis (1827), but the notation $\theta_{i}$ (or $\vartheta_{i}$ ), $i=0,1,2,3$, was introduced only in his 1837 lectures. Conferre [Ho], §11.) To get it exactly one has, among other things, to change the period from $2 \pi$ to 1 . Thus e.g. by definition

$$
\theta_{3}(z, \tau)=\sum_{n=-\infty}^{\infty} q^{n^{2}} e^{\pi i n x}
$$

where we, as is customary have written $q=e^{-\pi i t}$. For $s$ general, $\mathfrak{6}_{s}$ gives a generalization (call it "an $s$-generalization") $\mathfrak{6}_{s}$ of the Jacobi functions, which maybe has not yet been studied in the literature.

We conclude this section by writing down the following ad hoc generalization of Lions's formula [L2]:

$$
K_{s}(x, t ; b, a)=\int_{0}^{2 \pi} \Theta_{\frac{s}{2}}(x-y, t) \Theta_{\frac{s}{2}}(b-y, a) d y .
$$

(As in our previous discussion this follows immediately taking account of the orthogonality relations for the functions $e^{i n x}$.) A similar formula as (5.28) holds also in the non-periodic 
case of Section 5.4. But we suspect that it was maybe not the one that Lions himself had in mind in [L2] (in connection with his (4.15)).

5.6. The heat equation (general domain). It is easy to carry over the considerations of the previous Section 5.4 to any number of dimensions, also replacing the "cylinder" based on the interval $(0,2 \pi)$ by a general cylinder.

So let $\Omega$ be any bounded open set with smooth boundary $\Gamma$ in $\mathbb{R}^{\tau}$, say. (Because we want to have the letter $n$ free for denoting summation indices, we let the dimension be $\tau$.) Let us consider the cylinder $\Sigma=\Omega \times(0, \infty)$ with points $(x, t)$, with $x \in \Omega, t>0$. It will be convenient to use (as in [BS]), instead of minus the Laplacian $-\Delta$, the "smoothed" operator $-\Delta+q$, where $q$ is a smooth positive function in $\Omega$. Accordingly, we consider in $\Sigma$ the corresponding modified heat equation

$$
\frac{\partial u}{\partial t}=\Delta u-q u
$$

There is no reason to work with complex scalars, so let they again be real. Pick an orthonormal basis of the Hilbert space $L^{2}(\Omega)$ consisting of eigenfunctions of the operator $-\Delta+q$, say with Dirichlet boundary conditions. Let $\lambda_{n}$ be the corresponding eigenvalues (taken with their multiplicities), $-\Delta f_{n}+q f_{n}=\lambda_{n} f_{n}$. Note that in view of our assumption on $q$ we have $\lambda_{n}>0$. A solution $f$ of (5.29) ("a temperature") has then formally the Fourier expansion

$$
f(x, t)=\sum_{n} A_{n} f_{n}(x) e^{-\lambda_{n} t}
$$

Formally setting $t=0$ here we get for its trace (initial value):

$$
f(x, 0)=\sum_{n} A_{n} f_{n}(x)
$$

(The series in (5.30) and (5.31) are, apparently, "Minakshisundaram-Pleijel type" analogues of theta series; cf. the observation after (1.10).) We introduce then the Hilbert space $\mathscr{K}(\Sigma)$ of temperatures $f$ corresponding to the metric

$$
\|f\|_{s}^{2}=\sum_{n} \lambda_{n}^{s} A_{n}^{2}
$$

Running the same machinery once again one obtains the reproducing kernel

$$
K_{s}(x, t ; b, a)=\sum_{n} \lambda_{n}^{-s} f_{n}(x) f_{n}(b) e^{-(t+a) \lambda_{n}} .
$$

It is likewise manifest that the Lions type formula (5.28) extends mutatis mutandis to the kernel in (5.32). Indeed, let us introduce the elementary solution

$$
E(x, y ; t)=\sum_{n} f_{n}(x) f_{n}(y) e^{-t \lambda_{n}} \quad(x, y \in \Omega, t>0) .
$$

More generally, set 


$$
E_{s}(x, y ; t)=\sum_{n} \lambda_{n}^{-s} f_{n}(x) f_{n}(y) e^{-t \lambda_{n}} \quad(x, y \in \Omega, t>0) .
$$

Then one sees readily that

$$
K_{s}(x, t ; b, a)=\int_{\Omega} E_{\frac{s}{2}}(x, y ; t) E_{\frac{s}{2}}(b, y ; a) d y .
$$

Proof of (5.35). Indeed, the integrand of (5.35) can be written as

$$
\sum_{n} \lambda_{n}^{-\frac{s}{2}} f_{n}(x) f_{n}(y) e^{-t \lambda_{n}} \times \sum_{m} \lambda_{m}^{-\frac{s}{2}} f_{m}(b) f_{m}(y) e^{-a \lambda_{m}}
$$

which product we formally multiply together. In view of the orthogonality of $f_{n}$ and $f_{m}$, in the resulting double sum only the terms with $m=n$ give a contribution. Thus as a result we obtain the single sum

$$
\sum_{n} \lambda_{n}^{-s} f_{n}(x) f_{n}(b) e^{-(t+a) \lambda_{n}}
$$

which is the right hand side of (5.32).

\section{References}

[A] N. Aronszajn, Theory of reproducing kernels, Trans. Amer. Math. Soc. 68 (1950), 337-404.

[B] S. Bergman, The kernel function and conformal mapping, Second, revised edition, Math. Surv. 5, American Mathematical Society, Providence 1970.

[BS] S. Bergman, M. Schiffer, Kernel functions and elliptic differential equations in mathematical physics, Academic Press, New York 1953.

[EP] M. Engliš, J. Peetre, A Green's function for the annulus, Ann. Mat. Pura Appl. (IV) 171 (1996), $313-377$.

[Fo] G. B. Folland, Introduction to partial differential equations, Second edition, Princeton University Press, Princeton, New Jersey 1995.

[F] J. Fourier, The analytic theory of heat, Dover, New York 1955.

[FO] D. Fujiwara, S. Ozawa, The Hadamard variational formula for the Green function of some normal elliptic boundary problems, Proc. Japan Acad. Sci. 54 (1978), 215-220.

[G1] P. Garabedian, Schwarz's lemma and the Szegö kernel function, Trans. Amer. Math. Soc. 67 (1949), $1-35$.

[G2] P. Garabedian, Partial differential equations, Second edition, Chelsea, New York 1986.

$[\mathrm{GH}] \quad$ L. Greengard, J. Helsing, On the numerical evaluation of elastostatic fields in locally isotropic twodimensional composites, J. Mech. Phys. Solids 46 (1998), 1441-1462.

[H] J. Hadamard, Mémoire sur le problème d'analyse relatif à l'équilibre des plaques élastiques encastrées, Mém. Acad. Sci. Inst. France 33, no. 4 (1908), 128-254.

[H1] L. Hörmander, Linear partial differential equations, Grundl. math. Wiss. 116, Springer-Verlag, BerlinHeidelberg-New York 1968.

[H2] L. Hörmander, The analysis of linear partial differential operators, Grundl. math. Wiss. 256, 257, 274, 275, Springer-Verlag, Berlin-Heidelberg-New York-Tokyo 1985.

[Ho] C. Houzel, Fonctions elliptiques et intégrales abéliennes, in: J. Dieudonné, éd., Abregé d'histoire des mathématiques 1700-1900, Hermann, Paris (1978), 1-133.

[K] E. Kamke, Differentialgleichungen, Lösungsmethoden und Lösungen, I, Gewöhnliche Differentialgleichungen, 6. Auflage, Leipzig 1959; Russian translation: Nauka, Moscow 1971.

[L1] J.-L. Lions, Noyau reproduisants et système d'optimalité, in: Aspects of mathematics and its applications, J. A. Barroso, ed., North-Holland Math. Library 34, Elsevier, Amsterdam (1986), 573-582.

[L2] J.-L. Lions, Remarks on reproducing kernels of some function spaces, Function spaces, interpolation theory, and related topics (Lund 2000), A. Kufner, M. Cwikel, M. Engliš, L.-E. Persson, and G. Sparr, eds., Walter de Gruyter, Berlin (2002), 51-61. 
[LM] J.-L. Lions, E. Magenes, Problèmes aux limites non homogènes et applications, vol. 1, Dunod, Paris 1968.

[MOS] W. Magnus, F. Oberhettinger, R. P. Soni, Formulas and theorems for the functions of mathematical physics, Third edition, Grundl. math. Wiss. 52, Springer-Verlag, Berlin-Heidelberg-New York 1966.

[MP] S. Minakshisundaram, A. Pleijel, Some properties of the eigenfunctions of the Laplace-operator on Riemannian manifolds, Canadian J. Math. 1 (1949), 242-256.

[ML] Index of the Gösta Mittag-Leffler separate collection: Part I. Small boxes, Technical Report, Lund 1996, J. Peetre and T. Claeson, eds.; Big boxes. Part IIA (subjects), ibid. 2000, J. Peetre, ed.; Big boxes. Part IIB (authors), ibid. 2002, J. Peetre, ed.; http://www.maths.lth.se/matematiklu/personal/jaak.

[Pe1] J. Peetre, Théorèmes de regularité pour quelques classes d'opérateurs différentiels, Ph.D. thesis, Lund University, Sweden (1959), $122 \mathrm{pp}$.

[Pe2] J. Peetre, Elliptic partial differential equations of higher order, Lecture notes, University of Maryland (1962), 1-122.

[Pe3] J. Peetre, On Hadamard's variational formula, J. Diff. Equ. 36 (1980), 335-346.

[Pe4] J. Peetre, On Fourier's discovery of Fourier series and Fourier integrals, http://www.maths.lth.se/ matematiklu/personal/jaak. An abbreviated Swedish edition will appear in Normat.

[Sa] S. Saitoh, Theory of reproducing kernels and its applications, Pitman Res. Notes Math. Ser. 189, Longman Scientific \& Technical, Harlow 1988.

[Schi] M. Schiffer, The kernel of an orthogonal system, Duke Math. J. 13 (1945), 529-540.

[Schw] L. Schwartz, Sous-espaces hilbertiens d'espaces vectoriels topologiques et noyaux associés (noyaux reproduisant), J. Anal. Math. 13 (1964), 115-256.

[Sz] G. Szegö, Über orthogonale Polynome, die zu einer gegebenen Kurve der komplexen Ebene gehören, Math. Z. 9 (1921), 218-270.

[W] G. N. Watson, A treatise of the theory of Bessel functions, Cambridge University Press, Cambridge 1966.

[Z1] S. Zaremba, L'équation biharmonique et une classe remarquable de fonctions fondamentales harmoniques, Bull. Int. Acad. Sci. Cracov. (1907), 147-196; available also in the Mittag-Leffler separate collection (see $[\mathrm{ML}]$, Part I, p. 50).

[Z2] S. Zaremba, Sur le calcul numérique des fonctions demandées dans le problème de Dirichlet et le problème hydrodynamique, Bull. Int. Acad. Sci. Cracovie (1909), 125-195; available also in the Mittag-Leffler separate collection (see [ML], Part I, p. 50).

MÚ AV ČR, Zitná 25, 11567 Prague 1, Czech Republic e-mail: englis@math.cas.cz

Narvik University College, P.O. Box 385, 8505 Narvik, Norway e-mail: dl@hin.no

Centre for Mathematical Sciences, Lund University, Box 118, 22100 Lund, Sweden e-mail: jaak@maths.lth.se

Department of Mathematics, Luleå University of Technology, 97187 Luleå, Sweden e-mail: larserik@sm.luth.se

Eingegangen 12. Juli 2002 